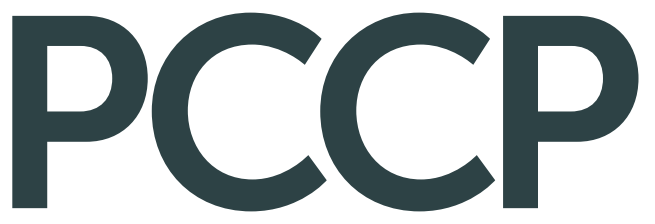

Physical Chemistry Chemical Physics www.rsc.org/pccp
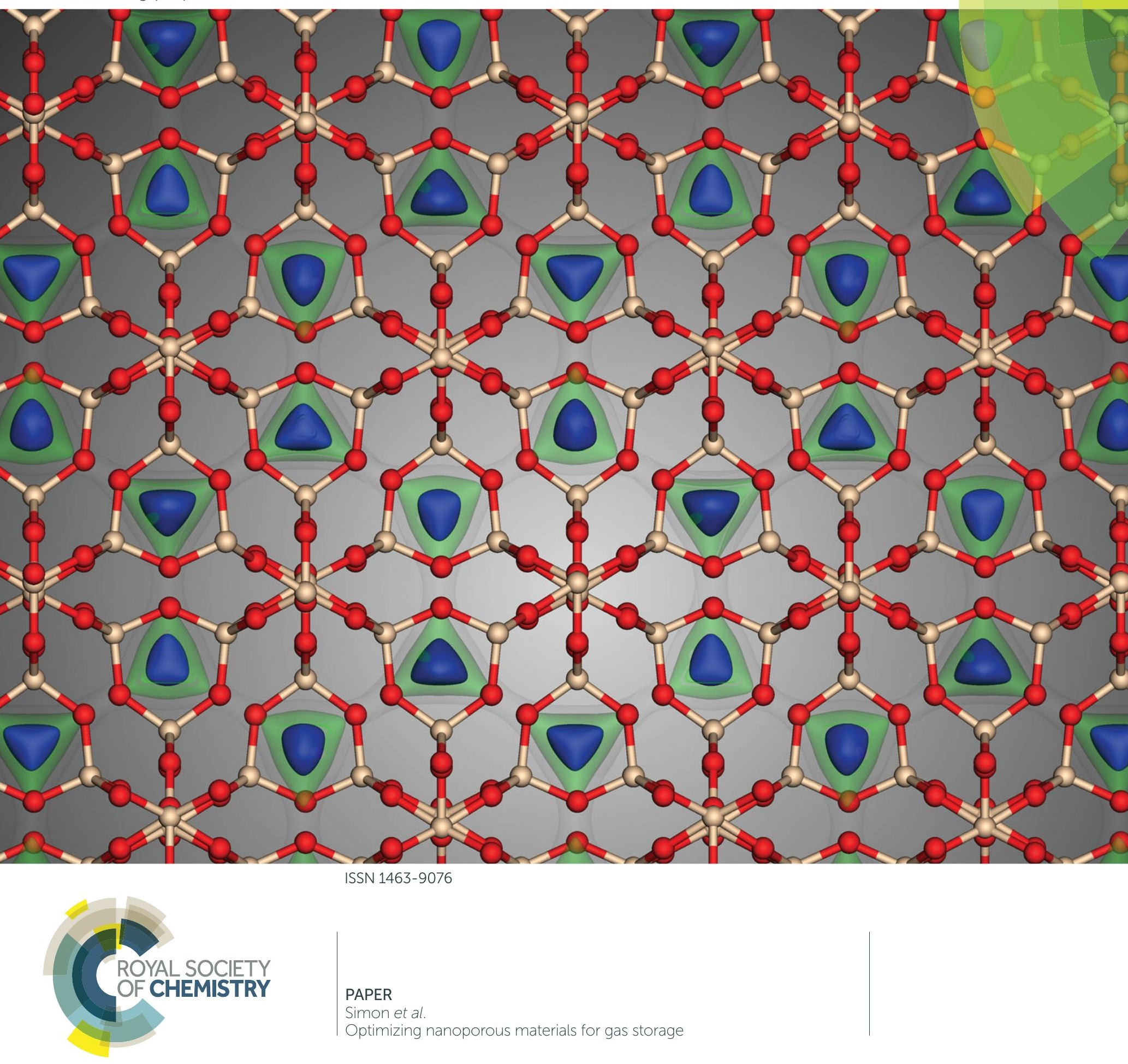
Cite this: Phys. Chem. Chem. Phys., 2014, 16, 5499

Received 30th August 2013, Accepted 3rd December 2013

DOI: $10.1039 / c 3 c p 55039 g$

www.rsc.org/pccp

\section{Optimizing nanoporous materials for gas storage $\dagger$}

\author{
Cory M. Simon, ${ }^{\text {a }}$ Jihan Kim, ${ }^{b}$ Li-Chiang Lin, ${ }^{a}$ Richard L. Martin, ${ }^{c}$ Maciej Haranczyk ${ }^{c}$ \\ and Berend Smit ${ }^{a}$
}

In this work, we address the question of which thermodynamic factors determine the deliverable capacity of methane in nanoporous materials. The deliverable capacity is one of the key factors that determines the performance of a material for methane storage in automotive fuel tanks. To obtain insights into how the molecular characteristics of a material are related to the deliverable capacity, we developed several statistical thermodynamic models. The predictions of these models are compared with the classical thermodynamics approach of Bhatia and Myers [Bhatia and Myers, Langmuir, 2005, 22, 1688] and with the results of molecular simulations in which we screen the International Zeolite Association (IZA) structure database and a hypothetical zeolite database of over 100000 structures. Both the simulations and our models do not support the rule of thumb that, for methane storage, one should aim for an optimal heat of adsorption of $18.8 \mathrm{~kJ} \mathrm{~mol}^{-1}$. Instead, our models show that one can identify an optimal heat of adsorption, but that this optimal heat of adsorption depends on the structure of the material and can range from 8 to $23 \mathrm{~kJ} \mathrm{~mol}^{-1}$. The different models we have developed are aimed to determine how this optimal heat of adsorption is related to the molecular structure of the material.

\section{Introduction}

Natural gas, composed mostly of methane, is an attractive fuel for transportation. Methane is the hydrocarbon with the greatest hydrogen to carbon ratio, endowing it with the least carbon dioxide emissions per energy harvested from its combustion. ${ }^{1}$ On an energy basis, emissions of $\mathrm{CO}_{2}$ from natural gas are $25 \%$ lower than those of gasoline. ${ }^{2}$ Since the transportation sector accounted for $28 \%$ of US greenhouse gas emissions in $2011,{ }^{3}$ replacing petroleum fuels with natural gas can make a substantial impact on mitigating climate change. Emissions in VOCs, $\mathrm{CO}, \mathrm{SO}_{x}$, and particulates ${ }^{4}$ are lower in natural gas than gasoline as well. Because methane is a potent greenhouse gas itself, these analyses are predicated on the minimization of leaks and releases that come with increased natural gas use. ${ }^{6}$

The economic incentives and goals to decrease dependency on oil enable the possibility of the mass-adoption of natural gasfueled vehicles. Abundant world-wide reserves of natural gas ${ }^{1}$ make the cost of natural gas competitive with gasoline. Recent technological advances in hydraulic fracturing and horizontal drilling sustain supply by making more natural gas in shale

\footnotetext{
${ }^{a}$ Department of Chemical and Biomolecular Engineering, University of California, Berkeley, California, USA.E-mail: CoryMSimon@gmail.com

${ }^{b}$ Department of Chemical and Biomolecular Engineering, Korea Advanced Institute of Science and Technology, 291 Daehak-ro Yuseong-gu, Daejeon, Korea 305-701

${ }^{c}$ Lawrence Berkeley National Laboratory, Berkeley, California, USA

$\dagger$ Electronic supplementary information (ESI) available. See DOI: 10.1039/ c3cp55039g
}

formations accessible for extracting ${ }^{7}$ - but not without its own costs (e.g., polluting drinking water). ${ }^{8}$ Recently, interest in methane hydrates, ${ }^{9}$ landfill gas, ${ }^{10}$ and livestock ${ }^{11}$ as potential sources of methane has been burgeoning. Further, in countries like the US, a vast, mature natural gas infrastructure is already in place ${ }^{5}$ facilitating the adoption of natural gas-fueled vehicles.

The technological obstacle to replacing liquid gasoline with natural gas is the comparatively low volumetric energy density of natural gas. While methane has a slightly larger gravimetric heat of combustion than gasoline, ${ }^{12}$ the inherent fact that methane is a gas at ambient conditions necessitates a densification strategy to obtain a competitive volumetric energy density. Currently, natural gas used by vehicles is densified for storage by either liquifaction at cryogenic temperatures (liquified natural gas, LNG) or compression to 200-300 bar at room temperature (compressed natural gas, $\mathrm{CNG}$ ), ${ }^{12}$ both of which require expensive infrastructure for fueling stations. CNG tanks require thick walls to endure the high pressures and a spherical or cylindrical geometry to evenly distribute stress. This results in a heavy, expensive tank that must be placed in the trunk. Similarly, LNG requires expensive cryogenic storage vessels.

A potential solution to enhance the volumetric energy density of methane with a less expensive and energy-intensive densification process is adsorbed natural gas technology. Van der Waals interaction forces between methane molecules and framework atoms of a nanoporous material, such as a zeolite or metalorganic framework, enable the physisorption of methane to yield a density greater than that of the gaseous phase at the 
same conditions. Packing a nanoporous material inside a natural gas fuel tank to physically adsorb methane gas at ambient temperatures would ideally require only moderate pressures (35-65 bar) to achieve the same volumetric energy density as CNG (200 bar). This would allow for lightweight, inexpensive, conformable vehicular fuel tanks. Another attractive feature of adsorbed natural gas tanks is the potential for home-refueling using incumbent natural gas pipelines since, compared to 200 bar CNG and liquefaction, relatively inexpensive two-stage compressors ${ }^{13}$ are required for refilling to $65 \mathrm{bar}$, mitigating infrastructure hindrances.

A large body of research ${ }^{13-17}$ aims to improve nanoporous materials for methane storage by modifying the framework chemistry, thereby tuning the interactions between the framework atoms and the methane. ${ }^{18}$ With the goal of achieving an energy that is competitive with gasoline using an adsorbent, we must maximize the quantity of methane stored in the material at the high pressure, $P_{\mathrm{H}}$, of a filled tank while, at the same time, minimizing the retention of gas at the low pressure, $P_{\mathrm{L}}$, of a depleted tank, i.e. the "cushion gas". The deliverable capacity or working capacity is defined as the difference in the loading in the adsorbent between the two pressures.

The Advanced Research Projects Agency-Energy (ARPA-E) of the US Department of Energy uses a target of $12.5 \mathrm{MJ} \mathrm{L}^{-1}$ deliverable capacity for natural gas sorbents ${ }^{19}$ at $298 \mathrm{~K}$ using a pressure range of 65 to 5.8 bar, which was recently modified from the original target pressure range of 35 to 1 bar. The discharge pressure was raised from 1 bar in recognition that a pressure differential must be present to drive a sufficient methane flow rate from the adsorbent to the engine. The charging pressure of $P_{\mathrm{H}}=65$ bar considers the threshold pressure for cheaper infrastructure at refilling stations and lighter-weight, conformable tanks. For comparison with the ARPA-E target, the volumetric energy density of CNG is $8.9 \mathrm{MJ} \mathrm{L}^{-1}$ (200 bar, $298 \mathrm{~K}$, Peng-Robinson equation of state) and of LNG is 22.2 $\mathrm{MJ} \mathrm{L}^{-1}\left(-161.5{ }^{\circ} \mathrm{C}\right)$, which is only $27 \%$ and $64 \%$ of gasoline (34.2 $\mathrm{MJ} \mathrm{L}^{-1}$ ), respectively. ${ }^{12}$ Using the energy of combustion of methane $\left(-889 \mathrm{~kJ} \mathrm{~mol}^{-1}\right)$, the ARPA-E target for adsorbed natural gas is $315 \mathrm{v}_{\mathrm{STP}} / \mathrm{v}$, where $\mathrm{v}$ denotes a unit of volume (this is nondimensional, so the units for volume are arbitrary). Once materials survive the $315 \mathrm{v}_{\mathrm{STP}} / \mathrm{v}$ deliverable capacity screening process, other properties must be considered, e.g., stability, good dynamic performance under fill-refill cycles, ${ }^{1,20}$ resistance to impurities, and cost. ARPA-E has also established a gravimetric deliverable capacity target, but the volumetric deliverable capacity is more important in determining the driving range from a tank fill-up. ${ }^{21}$

Here, we use a computational approach for a high-throughput screening of over 100000 zeolites from the hypothetical database generated by Deem and coworkers ${ }^{22,23}$ and the International Zeolite Association (IZA) database as adsorbent candidates for methane storage. While our screening study shows that zeolites are not particularly promising for exceeding ARPA-E targets, they serve here as a useful data set to investigate what properties endow a material with a large deliverable capacity, which is the goal of this work. Wilmer et $a l^{24}$ similarly explored geometrical relationships with methane storage capacity in a large number of hypothetical metal-organic frameworks (MOFs).
In addition, we aim to understand how the heat of adsorption affects the deliverable capacity. Bhatia and Myers ${ }^{25}$ were among the first to systematically study the relationship between the heat of adsorption and the deliverable capacity for a Langmuir isotherm. They noted that, to yield a high deliverable capacity, the affinity for methane in the adsorbent framework must be strong enough to store a large amount at the charging pressure $P_{\mathrm{H}}$, yet weak enough to release most of the methane at the discharge pressure $P_{\mathrm{L}} \cdot{ }^{25}$ Using experimental data for carbonaceous materials, Bhatia and Myers used classical thermodynamic arguments to demonstrate that the optimal heat of adsorption for methane storage is $18.8 \mathrm{~kJ} \mathrm{~mol}^{-1}$ for $P_{\mathrm{H}}=30$ and $P_{\mathrm{L}}=1.5$ bar. Their highly cited work provided much needed guidance, so much that the $18.8 \mathrm{~kJ} \mathrm{~mol}^{-1}$ target is now frequently used for a far wider class of materials than those that were envisioned in the original work of Bhatia and Myers. This raises the question of whether the assumptions underlying the work of Bhatia and Myers hold for such a wide class of materials.

Snurr and coworkers investigated the relation between the heat of adsorption and deliverable capacity by artificially changing the Lennard-Jones interaction parameters in a few select MOFs to tune the heat of adsorption in silico. ${ }^{26}$ They indeed observed an optimal heat of adsorption, but it was different for each material. In another work, ${ }^{27}$ they derive an upper bound for the optimal heat of adsorption by considering an adsorption site that results in complete loss of entropy of the adsorbate molecule. Garrone et al. ${ }^{28}$ pointed out that the entropy change upon adsorption is not the same for all materials and, using a positive correlation found between enthalpy and entropy changes upon adsorption, adjusted Bhatia and Myers' formula. In this work, we explain the enthalpy-entropy correlation and its consequences for designing the optimal material.

Our high-throughput, in silico screening facilitates our study of the relationship between material performance and key geometric and thermodynamic parameters. Moreover, to obtain a fundamental understanding of these relationships, we build a sequence of statistical mechanical models of adsorption.

\section{Zeolite screening data}

Here, we computationally screen 136328 hypothetical and 187 IZA zeolites for methane storage and investigate what properties endow zeolites with a high deliverable capacity.

\subsection{Methods}

The zeolite crystal structures were obtained from the SLC hypothetical database generated by Deem and coworkers. ${ }^{23} \mathrm{We}$ analyzed only structures with pore sizes accessible to methane (>1.625 A radius). Geometrical properties of the zeolite structures, such as accessible surface area and largest-included-sphere pore diameters are obtained using a Voronoi decomposition approach in the Zeo++ software. ${ }^{29}$ For every structure, we computed the equilibrium number of methane guest molecules per unit crystal cell as a function of temperature and chemical potential 
(related to pressure) with the grand-canonical Monte Carlo Algorithm. ${ }^{30}$ A Lennard-Jones potential models the frameworkguest and guest-guest interactions using force field parameters developed in ref. 31 and 32 . In the ESI, $\dagger$ Section S2, we provide a short review validating the force field developed in ref. 31 and 32, which was specifically tuned to reproduce the adsorption isotherms of methane and other alkanes in zeolites. To mimic an infinite crystal, we implement periodic boundary conditions with a shifted and truncated Lennard-Jones potential with a 12.0 A cutoff. For identification of top performing materials, we use the Peng-Robinson equation of state to convert fugacity to the experimentally relevant mechanical pressure.

We take into account in our simulations that some zeolites have adsorption pockets that can accommodate a methane molecule, but are not accessible from the gas phase. In our Monte Carlo simulations, we insert molecules at random positions and we therefore need to block these inaccessible sites to ensure that they are not sampled. In most conventional simulations, such pockets are identified by visual inspection. For screening large databases, we have developed a high-performance algorithm ${ }^{33,34}$ for this task. We segment the void space in the material into pockets (inaccessible regions of void space) and channels by inspecting the three-dimensional potential energy profile of methane in the material, computed prior to Monte Carlo sampling for speed-up of energy computations. We assume that the guest molecule is not able to cross an energetic barrier greater than $15 k_{\mathrm{B}} T$ and perform a multicore CPU flood fill process to segment the energy grid with respect to this barrier. We then identify how these segments are connected periodically, which affords the use of a convenient definition of a channel - a channel is a region of space from which you can travel to the equivalent region in another unit cell; the complement must be a pocket. Grid points within pockets are reassigned to a very large energy value, such that when Monte Carlo moves land within a pocket, they will experience a very large repulsive energy and be rejected, just as if the move had landed within an atom of the material.

The isosteric heat of adsorption is defined as the negative of the enthalpy change upon adsorption $\Delta H$. The energy of adsorption differs from the enthlapy of adsorption by a work term $(\Delta H=\Delta U-R T) .{ }^{35}$ We perform Widom insertions ${ }^{30}$ to obtain the ensemble average energy of adsorption at infinite dilution ( $U_{0}$ in following equations) and the Henry coefficient.

Adsorption isotherms, Henry coefficients, heats of adsorption, and geometric data of each structure are available at nanoporousmaterials.org/methanestorage, $\dagger$ including a list of the top 50 performers in both the 65 to 5.8 bar and 35 to 1 bar pressure ranges. In the scatter plots that follow, each zeolite structure is plotted as a point.

We point out that our molecular simulations, which implement periodic boundary conditions, predict the uptake in a perfect single crystal. Ultimately, the adsorbent in the final methane storage tank, which is not a single crystal, will depend upon the bulk adsorbent properties. For an adsorbent with a high-pressure uptake greater than free space, the deliverable capacity of the final, bulk adsorbent will necessarily be less than that of the single crystal; hence, our reported single crystal deliverable capacities of high-performing materials are an upper bound for the deliverable capacity of an adsorbed natural gas tank. However, the ARPA-E target of $315 \mathrm{v}_{\mathrm{STP}} / \mathrm{v}$ anticipates this and applies to the single crystal deliverable capacity. ${ }^{19}$

\subsection{Results}

We obtain adsorption isotherms at $298 \mathrm{~K}$ from 1 to $200 \mathrm{bar}$ from Grand-canonical Monte Carlo simulations. We used these to plot the deliverable capacity against loading at $P_{\mathrm{H}}=65$ bar in Fig. 1(a). To compare with using a free-space tank, the deliverable capacity and density at 65 bar of an ideal gas is plotted as horizontal and vertical lines, respectively.

Fig. 1(a) illustrates that a high loading is necessary, but not sufficient, for a high deliverable capacity. Two materials with the same high-pressure loading can exhibit substantially different deliverable capacities depending on the shape of the isotherm (see Fig. 1(b)). As an example, structure PCOD8294531 (red star) has a promising 65 bar loading of $163 \mathrm{v}_{\mathrm{STP}} / \mathrm{v}$. However, there is still so much methane left at the discharge pressure $P_{\mathrm{L}}=5.8 \mathrm{bar}$ that it exhibits a deliverable capacity of only $33 \%$ of this, rendering it useless (a free-space tank is better). This highlights the importance to consider more than the absolute storage capacity at 65 bar.

Next, we investigate relationships between deliverable capacity and geometrical properties. First, we explore if the size of the adsorption pore affects the deliverable capacity in Fig. 1(c). We characterize the largest pore size by assigning radii to the framework atoms, modeling them as hard-spheres, and finding the largest sphere that can be included in the framework without overlapping with a framework atom. Generally, there appears to be a distinct optimal largest pore size for both 65 and 5.8 bar loading (see ESI, $\dagger$ Fig. S7). As a result, structures with the highest deliverable capacities generally occur at an optimal pore size range around $4.6 \AA$. A second peak occurs around twice 4.6 $\AA$, likely corresponding to more than one methane molecule fitting inside the pore. Pore diameter is not, however, a good predictor of deliverable capacity and thus other variables must be considered. Second, we corroborate claims in the literature ${ }^{37}$ that high surface area polymeric materials result in high uptake with the positive correlation present in Fig. 1(d). Based on the colorcoding in Fig. 1(d), a material can somewhat compensate for its lack of accessible surface area by achieving a high heat of adsorption. Because high uptake is a prerequisite for high deliverable capacity, there is also a positive correlation between the deliverable capacity and the surface area, but the relationship with the heat of adsorption is more complicated (see ESI, $\dagger$ Fig. S8).

One of the important predictions of the thermodynamic analysis of Bhatia and Myers is that there is an optimal heat of adsorption for given operating pressures. Using the assumptions in ref. 25 that the standard entropy change upon adsorption for methane is $\Delta S^{\circ}=-9.5 R$ and our relevant operating pressures $P_{\mathrm{H}}=65 \mathrm{bar}$ and $P_{\mathrm{L}}=5.8 \mathrm{bar}$, we expect a heat of adsorption of $16.2 \mathrm{~kJ} \mathrm{~mol}^{-1}$, plotted as a vertical line in Fig. 2, to yield materials with the highest deliverable capacity. We plot the deliverable capacity against the heat of adsorption in Fig. 2. However, materials endowed with a heat of adsorption of $16.2 \mathrm{~kJ} \mathrm{~mol}^{-1}$ exhibit a 


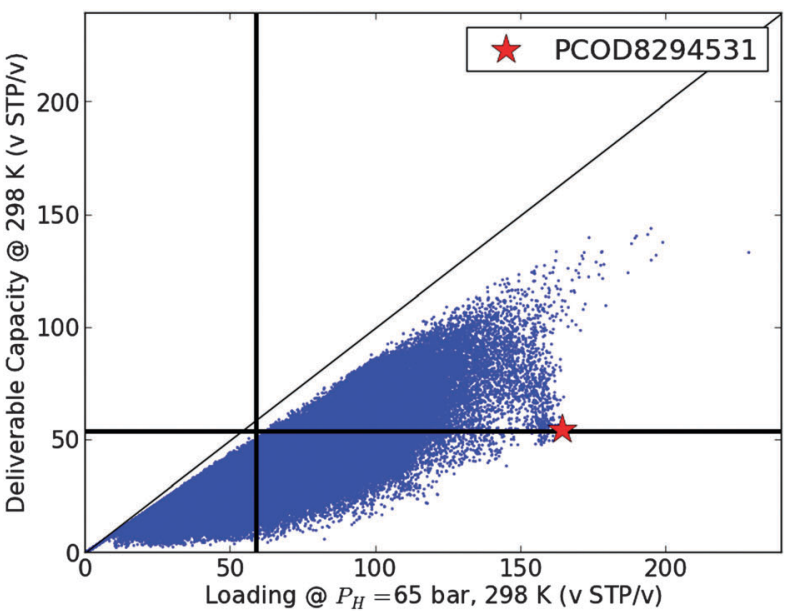

(a)

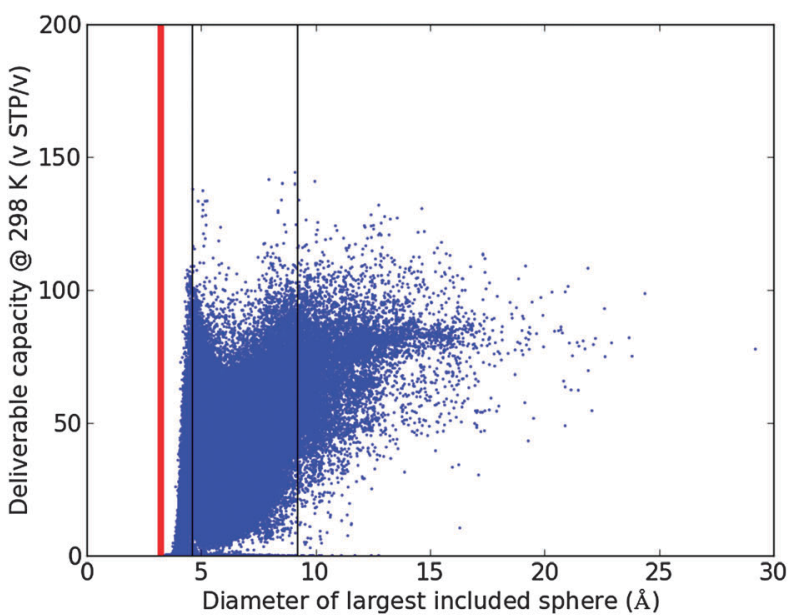

(c)

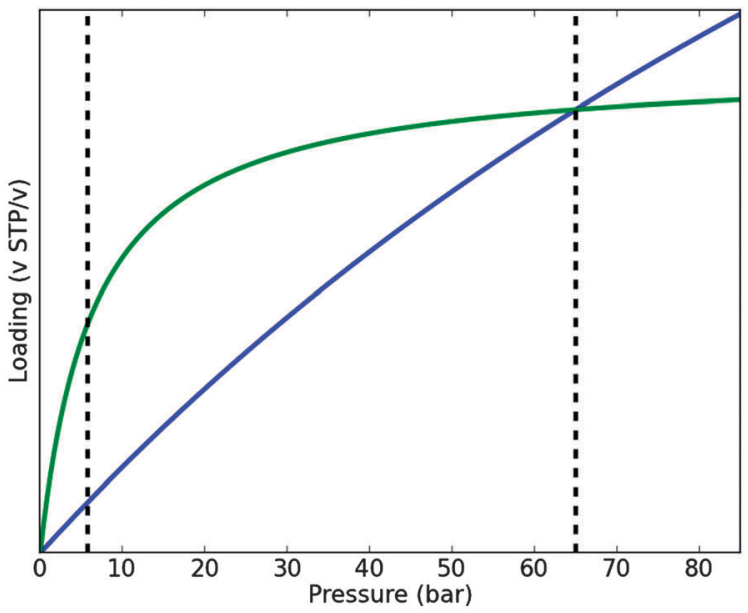

(b)

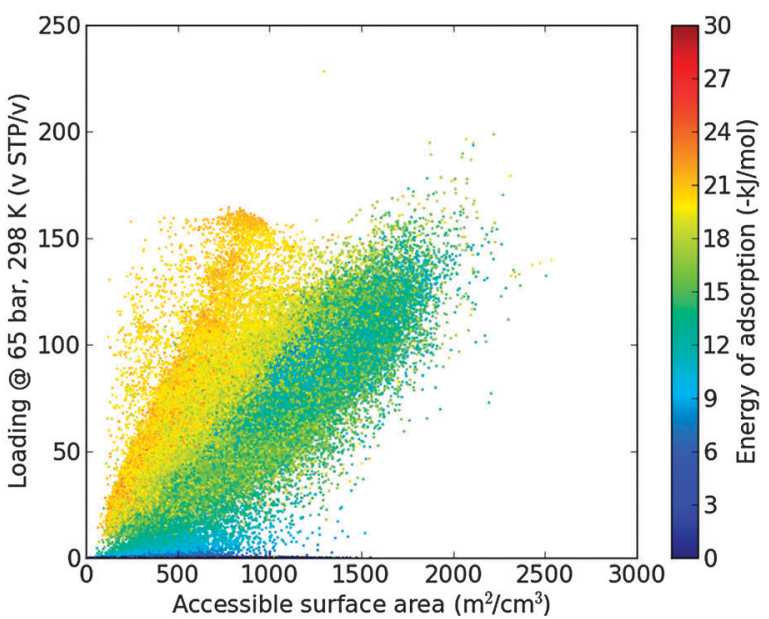

(d)

Fig. 1 (a) Deliverable capacity of structures in the zeolite database against high-pressure loading. The cushion gas (loading at 5.8 bar) can significantly degrade the deliverable capacity from the high-pressure loading. Vertical line: ideal gas density at 65 bar $(58.76$ VSTP/v). Horizontal line: deliverable capacity of free space tank (ideal gas, $53.52 \mathrm{v}_{\mathrm{STP}} / \mathrm{v}$ ). (b) Depending on isotherm shape, two materials with the same high-pressure loading can have very different deliverable capacities. The green isotherm saturates quickly, resulting in a high uptake at the low pressure as well, and thus lower deliverable capacity. (c) Deliverable capacity plotted against the largest included sphere diameter. Red vertical line is hard-sphere diameter of methane, $3.25 \AA .{ }^{36}$ Black vertical lines denote $4.6 \AA$ and $2 \times 4.6=9.2 \AA$. (d) Loading at 65 bar vs. accessible surface area color-coded according to the energy of adsorption.

drastic range of deliverable capacities. Further, there are many high-performance materials with heats of adsorption far from the optimal. To try to better understand this, we color-code the points in Fig. 2 by the void fraction of the material. To an extent, the void fraction helps distinguish deliverable capacities between materials with the same heat of adsorption; a larger void fraction, implying a more porous material, generally results in a higher deliverable capacity. To understand the discrepancy between Fig. 2 and the concept of a blanket optimum heat of adsorption, we proceed to develop a series of statistical mechanical models of adsorption.

\section{Models for adsorption: seeking an optimal heat of adsorption}

We develop a series of models to quantify and intuit how the heat of adsorption affects the deliverable capacity. We start with a simple model (model 0), which is constructed such that the heat of adsorption is independent of the structure of the material. As in this model the entropy can be varied independently, we obtain insight into the role of entropy in determining the optimal adsorption conditions. In model 0 , we assume that the gas molecules do not interact; in model 1 we study the effect of gas-gas interactions on the performance of the materials. In model 2, we further study the correlation between heat of adsorption and entropy of adsorption that has been found in experimental studies. Model 3, in ESI, $\dagger$ Section S7, similarly explores how a heterogeneous energy landscape is linked to the entropy of adsorption.

\subsection{Model 0: Langmuirian material}

In model 0 , our idealization of a nanoporous crystalline material is that it consists of $M$ identical, independent adsorption sites in 


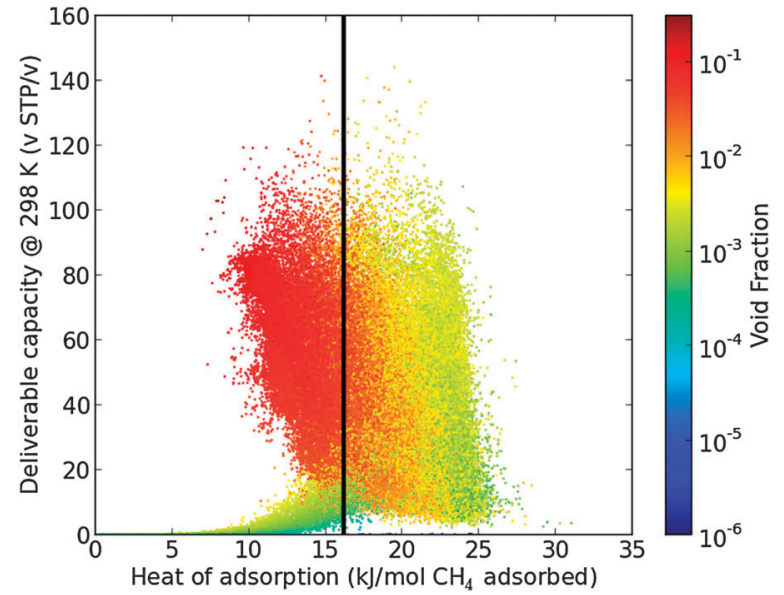

Fig. 2 Deliverable capacity vs. heat of adsorption color-coded according to the void fraction. Vertical line denotes the optimal heat of adsorption in eqn (5) using the assumption $\Delta S^{\circ}=-9.5 R$ and a pressure range of 65 to $5.8 \mathrm{bar}, 16.2 \mathrm{~kJ} \mathrm{~mol}^{-1}$.

a unit volume. Each crystalline unit cell has a void space $\Omega_{\mathrm{s}}$ that is of uniform energy $U_{0}$ and large enough for only one gas molecule. The region outside the binding site is inaccessible because it is occupied by framework atoms. See Fig. 3. The energy landscape of the material is thus:

$$
U(x)= \begin{cases}U_{0}, & x \in \Omega_{\mathrm{s}} \\ \infty, & x \notin \Omega_{\mathrm{s}} .\end{cases}
$$

With this energy landscape, we derive in the ESI, $\dagger \mathrm{S} 4$ from statistical mechanics the isotherm for this model material under the assumption that nearby guests do not interact.

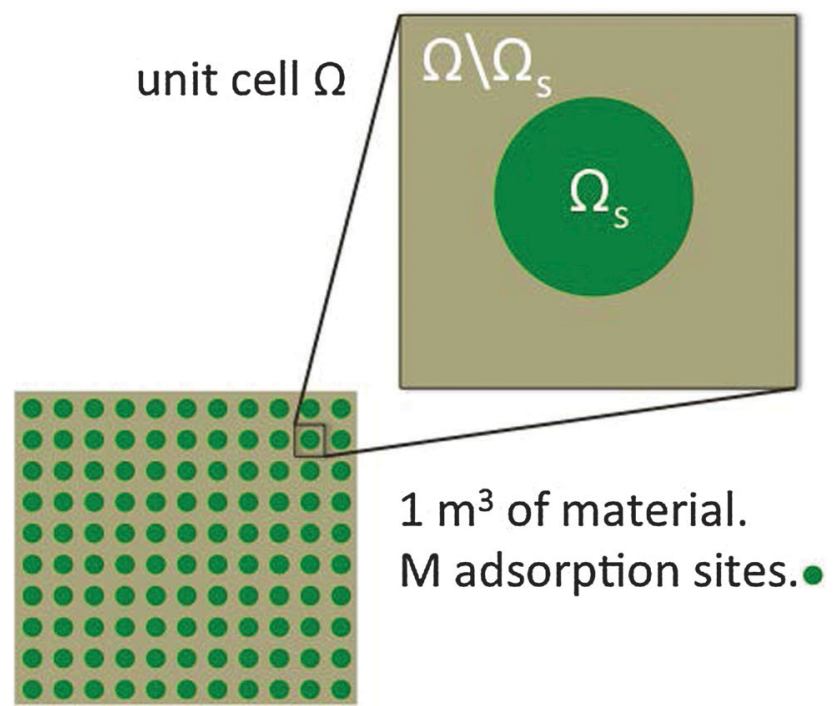

Fig. 3 Our model material. Crystalline unit cell consists of binding site $\Omega_{\mathrm{s}}$ (green) that can be occupied by one guest molecule. The region outside the binding pocket (brown) is unoccupiable due to the presence of framework atoms. The bulk crystal is composed of $M$ unit cells per volume, endowing it with $M$ adsorption sites per unit volume.
The adsorbed guest loading (moles or molecules per volume) $\sigma$ in the material at pressure $P$ follows a Langmuir isotherm:

$$
\sigma=\frac{\beta \varepsilon \mathrm{e}^{-\beta U_{0} P}}{1+\frac{\beta \varepsilon \mathrm{e}^{-\beta U_{0}}}{M} P},
$$

where $\varepsilon$ is the void fraction, or porosity, of the material $\left(\varepsilon=\left|\Omega_{\mathrm{s}}\right| /|\Omega|\right)$ and $\beta=1 /(R T)$ with $R$ as the universal gas constant. Eqn (2) is the familiar Langmuir isotherm with Henry coefficient $K_{\mathrm{H}}=\beta \varepsilon \mathrm{e}^{-\beta U_{0}}$ and Langmuir constant $K=\frac{\beta \varepsilon \mathrm{e}^{-\beta U_{0}}}{M}$.

The isothermal deliverable capacity $D$ of a material is the difference in loading at the high pressure $P_{\mathrm{H}}$ of a filled tank and the low pressure $P_{\mathrm{L}}$ of a depleted tank. Using our model, the methane delivered to the engine per volume of model material is:

$$
D\left(U_{0} ; P_{\mathrm{L}}, P_{\mathrm{H}}\right)=\frac{\beta \varepsilon \mathrm{e}^{-\beta U_{0}} P_{\mathrm{H}}}{1+\frac{\beta \varepsilon \mathrm{e}^{-\beta U_{0}}}{M} P_{\mathrm{H}}}-\frac{\beta \varepsilon \mathrm{e}^{-\beta U_{0}} P_{\mathrm{L}}}{1+\frac{\beta \mathrm{e}^{-\beta U_{0}}}{M} P_{\mathrm{L}}},
$$

which is lucidly a function of the energy of adsorption $U_{0}$. The two terms show the prerequisite to obtain a large deliverable capacity is a high uptake at $P_{\mathrm{H}}$, but uptake at the depletion pressure $P_{\mathrm{L}}$ reduces the deliverable capacity below the highpressure loading (see Fig. 1(a)).

To search for a $U_{0}$ that optimizes the deliverable capacity, we solve the eqn $\frac{\partial D}{\partial U_{0}}=0$ and find:

$$
U_{0, \mathrm{opt}}=U_{0, \mathrm{opt}}\left(\frac{\varepsilon}{M}\right)=R T \ln \left(\frac{\sqrt{P_{\mathrm{L}} P_{\mathrm{H}}}}{R T} \frac{\varepsilon}{M}\right) .
$$

The optimum enthalpy of adsorption is then $\Delta H_{\mathrm{opt}}=U_{0, \mathrm{opt}}-R T$. Bhatia and Myers' derivation from classical thermodynamics yields

$$
\Delta H_{\mathrm{BM}}=\frac{R T}{2} \log \left(\frac{P_{\mathrm{L}} P_{\mathrm{H}}}{\left(P^{\circ}\right)^{2}}\right)+T \Delta S^{\circ},
$$

where $\Delta S^{\circ}$ is the standard entropy change upon adsorption with reference pressure $P_{0}=1$ bar. ${ }^{38}$ Bhatia and Myers show that, for activated carbons and carbon nanotubes, the entropic term is roughly the same, and they obtain their optimal heat of adsorption by using $\Delta S^{\circ}=-9.5 R$ in eqn (5). If we assume $\Delta S^{\circ}=$ $-9.5 R$ holds for all materials, we obtain an optimal heat of adsorption for methane storage of $16.2 \mathrm{~kJ} \mathrm{~mol}^{-1}$ at ambient temperature with $P_{\mathrm{H}}=65$ bar and $P_{\mathrm{L}}=5.8$ bar. As Bhatia and Myers assume that the isotherms are described with a Langmuir model, $U_{0, \text { opt }}$ for our model 0 is consistent with eqn (5) (see $\mathrm{ESI}, \dagger \mathrm{S} 4.3)$. In addition, our model links the entropic term to the structural properties in model 0 , manifesting precisely how this term differs among materials.

Plugging $U_{0, \text { opt }}$ into eqn (3), we get the best possible deliverable capacity of our model material:

$$
D_{\text {opt }}\left(P_{\mathrm{L}}, P_{\mathrm{H}}\right)=M\left(\frac{P_{\mathrm{H}}}{\sqrt{P_{\mathrm{L}} P_{\mathrm{H}}}+P_{\mathrm{H}}}-\frac{P_{\mathrm{L}}}{\sqrt{P_{\mathrm{L}} P_{\mathrm{H}}}+P_{\mathrm{L}}}\right),
$$

equivalent to the maximum delivery obtained by optimizing the Langmuir constant in ref. 39. At extreme operating conditions,

$$
\lim _{P_{\mathrm{H}} \rightarrow \infty} D_{\mathrm{opt}}\left(P_{\mathrm{L}}=0, P_{\mathrm{H}}\right)=M,
$$


and each adsorption site will be fully utilized and deliver a methane molecule to the engine or fuel cell. The two previous equations bound the deliverable capacity below the number of adsorption sites per unit volume - and tuning the heat of adsorption does not change this. The heat of adsorption optimizes the deliverable capacity for a fixed number of sites $M$. More precisely, it maximizes the fraction of sites $\left(\frac{D}{M}\right)$ in a given material that can deliver a methane molecule. ${ }^{39}$ The density of adsorption sites $M$ is thus important in obtaining a high deliverable capacity, and from here one can tune the material's chemistry to obtain the optimal energetics. The analysis in ref. 25 aimed to demonstrate that for a fixed maximum loading, there exists an optimal heat of adsorption. This partially explains the discrepancy between Fig. 2, where the heat of adsorption is not a good predictor of deliverable capacity, and the rule of thumb in the literature that a heat of adsorption of $18.8 \mathrm{~kJ} \mathrm{~mol}^{-1}$ provides an optimum material for methane storage.

Next, we selected $\sim 2500$ structures identified as Langmuirian by our isotherm fitting routine (see ESI, $\dagger$ S8) to analyze in the context of model 0 . As a criterion, we use the residual normalized by the predicted maximum loading from the Langmuir fitting routine. We selected only the zeolites that have a good Langmuir model fit because (i) model 0 applies to only Langmuirian materials; and (ii) we want to ensure confidence in the accuracy of identification of $M$ from our high-throughput data fitting routine. In Fig. S5 (ESI $\dagger$ ), we show that this set of selected materials explores a vast range of geometric parameters and heats of adsorption and hence can be considered a diverse set. In Fig. 4 (top, blue points), we plot the fractional deliverable

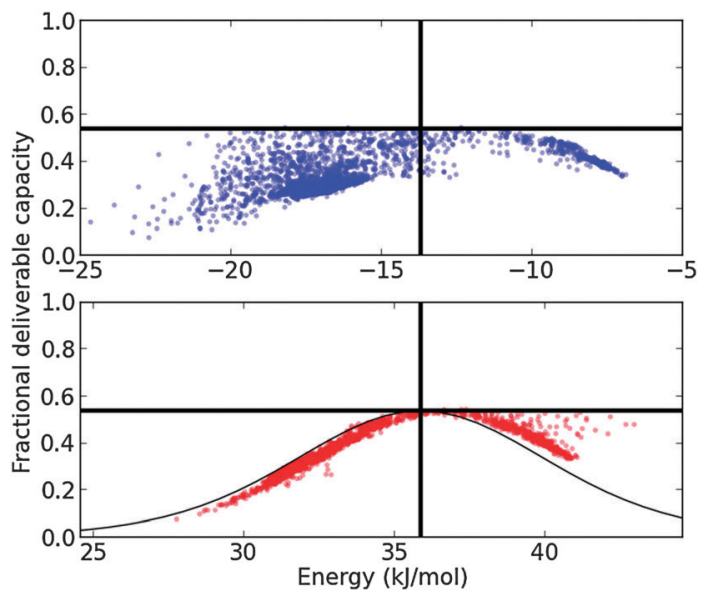

Fig. 4 Fractional deliverable capacity plots for Langmuirian structures. Horizontal line: maximum theoretical fractional deliverable capacity in eqn (6). (top) The $x$-axis is the infinite dilution energy of adsorption $U_{0}$ computed from simulation. Vertical line: predicted optimal energy of adsorption, $-13.7 \mathrm{~kJ} \mathrm{~mol}^{-1}$, using Bhatia and Myers' assumption of $\Delta S^{\circ}=$ $-9.5 R$, corresponding to an enthalpy of adsorption of $-16.2 \mathrm{~kJ} \mathrm{~mol}^{-1}$. (bottom) The $x$-axis is the effective energy of adsorption $U^{*}$ in eqn (8). Vertical line: $U_{\text {opt }^{*}}=\frac{R T}{2} \log \left(P_{\mathrm{H}} P_{\mathrm{L}}\right)$. The black curve is the theoretical universal curve (ESI, $\dagger$ S4.2), a function of the effective energy of adsorption, on which all materials should fall. capacity as a function of the energy of adsorption for these Langmuirian materials. Also for the fractional deliverable capacity, we do not see that the best structures have the predicted optimal heat of adsorption of $16.2 \mathrm{~kJ} \mathrm{~mol}^{-1}$. Further, several structures are endowed with a heat of adsorption of $16.2 \mathrm{~kJ} \mathrm{~mol}^{-1}$, but do not have the maximum fractional deliverable capacity in eqn (6). The reason is that the assumption in ref. 25 that the entropy of adsorption is constant may not hold, implying that the optimal heat of adsorption varies from material to material. This is evident from eqn (4) where the structural characteristic $\frac{\varepsilon}{M}$ appears, which is the volume of the adsorption pore.

The standard entropy loss upon adsorption is related to the volume of the adsorption site, $\frac{\varepsilon}{M}$ (see ESI, $\uparrow \mathrm{S} 4.3$ ). For the zeolite structures, we estimate the void fraction using an energetic approach, where points in space that have less potential energy than a threshold distance from the ensemble average energy are deemed occupiable (see ESI, $\dagger$ S1). Fig. 5(a) is an $M-\varepsilon$ scatter plot for the Langmuirian materials in the zeolite database. If the entropic change upon adsorption were the same for all zeolites, all points would collapse to a single line. The color-coding illustrates that we have many materials with different $\frac{\varepsilon}{M}$ and thus different entropic changes upon adsorption. To see the relevant range of $\frac{\varepsilon}{M}$ experienced in the zeolite database, which will dictate the range of the optimal heat of adsorption via eqn (4), we take the range of the inverse slopes in Fig. 5(a). Also note that $\varepsilon$ and $M$ have a weak correlation; if a material has a large void fraction $\varepsilon$, it may be a result of having many adsorption sites.

Model 0 predicts that if a material has a different $\frac{\varepsilon}{M}$ (related to $\Delta S^{\circ}$ ), it has a different optimal heat of adsorption. In Fig. 5(b), we quantify this effect for the range of $\frac{\varepsilon}{M}$ we observed in the zeolite database in Fig. 5(a). Fig. 5(b) shows that as the adsorption site decreases in volume, corresponding to a larger entropic penalty upon adsorption, the optimal heat of adsorption increases to compensate for the entropic loss. Depending on the $\frac{\varepsilon}{M}$, the optimal heat of adsorption ranges from 11 to $22 \mathrm{~kJ} \mathrm{~mol}^{-1}$.

According to our model, all materials will fall on a universal curve when we subtract off the entropic dependence in $U_{0 \text {,opt }}$ and define an effective energy of adsorption:

$$
U^{*}:=U_{0}-R T \ln \left(\frac{1}{R T} \frac{\varepsilon}{M}\right)
$$

We derive the universal curve in the ESI, $\dagger \mathrm{S} 4.2$ and plot it as a black curve in Fig. 4 (bottom). Now, the theoretical optimal effective energy of adsorption is $U_{\text {opt }}{ }^{*}=\frac{R T}{2} \log \left(P_{\mathrm{L}} P_{\mathrm{H}}\right)$, constant for every material. To test our model, we plot the fractional deliverable capacity against the effective energy of adsorption in Fig. 4 (bottom, red points) along with $U_{\text {opt }}{ }^{*}$ as a vertical line. The cloud of blue points collapses onto a near universal curve when 


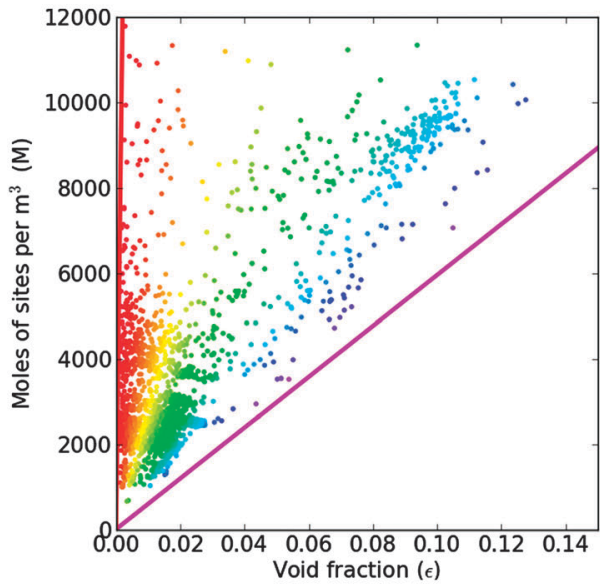

(a)

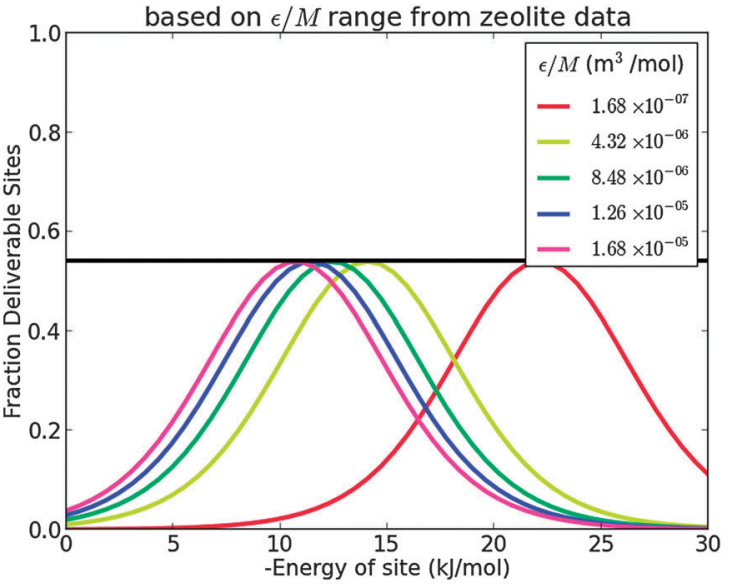

(b)

Fig. 5 (a) The void fraction $\varepsilon$ and number of sites $M$ are correlated in the Langmuirian materials, but $\frac{\varepsilon}{M}$ (inverse slope here) is constrained to a range. Points are color-coded according to their $\frac{\varepsilon}{M}$ value. The inverse slopes of the two solid lines (pink, red) at the boundary of the point cloud are the extrema in the set of $\frac{\varepsilon}{M}$ in the zeolite database. (b) The optimum energy of adsorption $U_{0}$ is a function of $\frac{\varepsilon}{M}$. The range of $\frac{\varepsilon}{M}$ is taken from the slopes in (a) and the colors of the curves correspond to (a).

we consider the effect of differing $\frac{\varepsilon}{M}$ among the materials. The universal curve corresponds to the theoretical predictions. Knowledge of $\frac{\varepsilon}{M}$ and the heat of adsorption is thus enough to make a reasonable prediction of the fractional deliverable capacity for Langmuirian materials. The slight shift of the red points from the theoretical universal curve is explained from model 3 in the ESI. $\dagger$

\subsection{Model 1: incorporating guest-guest interactions}

Model 1 builds upon model 0 to include the feature of guestguest interactions. A reasonable abstraction of adsorbed gas molecules interacting with each other is that each nearby guest enhances the energy of a free adsorption site by energy $U_{\mathrm{gg}}$ (subscripts for "guest-guest"). Using a mean-field approximation, the energy landscape is now a function of the number of occupied adsorption sites:

$$
U(x)= \begin{cases}U_{0}+z \frac{\sigma}{M} U_{\mathrm{gg}}, & x \in \Omega_{\mathrm{s}} \\ \infty, & x \notin \Omega_{\mathrm{s}} .\end{cases}
$$

The energy of each site at infinite dilution, without any guest-guest interactions, is $U_{0}$, and $z$ is the coordination number around a site (e.g., $z=6$ in $\mathbb{R}^{3}$ for a cubic lattice). In the ESI, $\dagger \mathrm{S} 5$, we derive the self-consistency equation for our mean field model material with the energy landscape in eqn (9):

$$
\sigma=\frac{\beta \varepsilon \mathrm{e}^{-\beta U_{0}} \mathrm{e}^{-\beta U_{\mathrm{gg}} z \sigma / M} P}{1+\frac{\beta \mathrm{e}^{-\beta U_{0}}}{M} \mathrm{e}^{-\beta U_{\mathrm{gg} z \sigma / M} P} .}
$$

We can intuit this in the context of a traditional Langmuir isotherm with an effective Langmuir constant $\hat{K}:=K \mathrm{e}^{-\theta \sigma / M}$, where $\theta:=\beta U_{\mathrm{gg}} z$ is a guest-guest interaction dependent term and $K:=\frac{\beta \varepsilon \mathrm{e}^{-\beta U_{0}}}{M}$ as in model 0 . That is, now the Langmuir constant is a function of loading. The effect is that, for attractive guest-guest interactions $\left(U_{\mathrm{gg}}<0\right)$, the effective Langmuir constant increases as loading increases. As the solid loads with adsorbates upon increasing the pressure, the loading lies on a Langmuir isotherm with a larger and larger Langmuir constant. The opposite is true for repulsive guest-guest interactions. To gain further insight, we perform an asymptotic expansion in $\theta$ (ESI, $\uparrow$ S5.2) to obtain the loading in eqn (10) as a correction to a Langmuir isotherm:

$$
\frac{\sigma}{M} \sim \frac{K P}{1+K P}+\theta\left(\frac{K P}{1+K P}\right)^{2}\left(\frac{K P}{1+K P}-1\right) .
$$

The correction term goes to zero as both $P \rightarrow 0$ and $P \rightarrow \infty$, consistent with our expectation that guest-guest interactions cause a negligible deviation from a Langmuir isotherm at low and high enough pressures. At low pressures, and hence dilute loading, there are very few nearby guests for interaction. At high enough pressures, there is enough chemical potential driving force such that each site is occupied, regardless of its energy, and the loading is near $M$. We plot in Fig. 6(a) the numerical solution to eqn (10) with $\theta= \pm 0.5$ to represent repulsive and attractive guest-guest interactions, respectively, to visualize the deviation from a Langmuir isotherm. The asymptotic approximation to the solution in eqn (11), shown with the dashed line, is in excellent agreement with the numerical solution to eqn (10).

From the behavior of the correction term in eqn (11), guestguest interactions can benefit the deliverable capacity of a material by substantially increasing loading at $P_{\mathrm{H}}$, but not the loading at $P_{\mathrm{L}}$. If the isotherm begins to saturate before $P_{\mathrm{L}}$, however, guest-guest interactions can degrade the deliverable capacity by increasing uptake at $P_{\mathrm{L}}$ more than $P_{\mathrm{H}}$. See ESI, $\dagger$ Fig. S3(a) for illustrative sketches. 


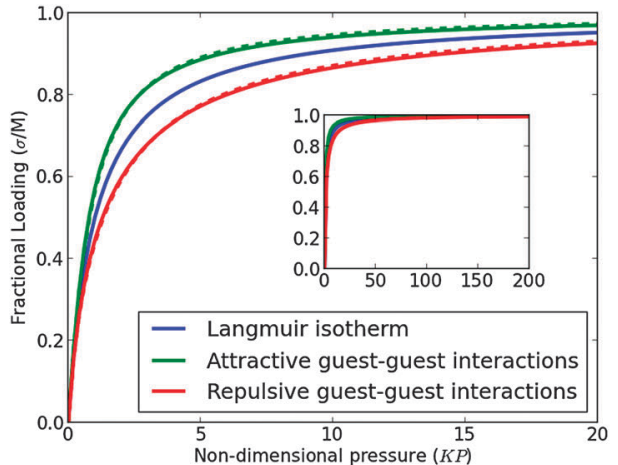

(a)

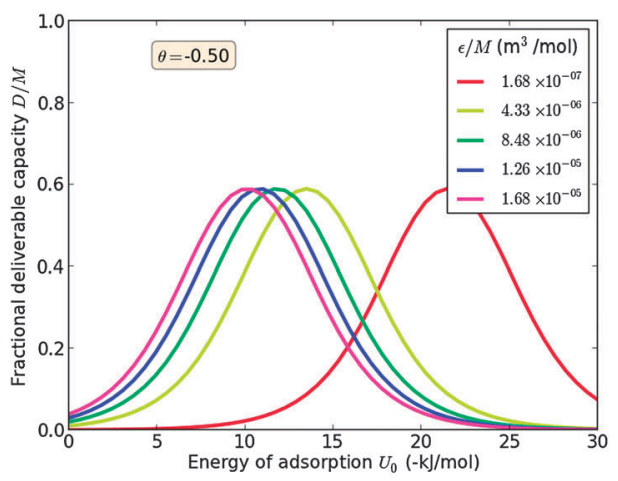

(c)

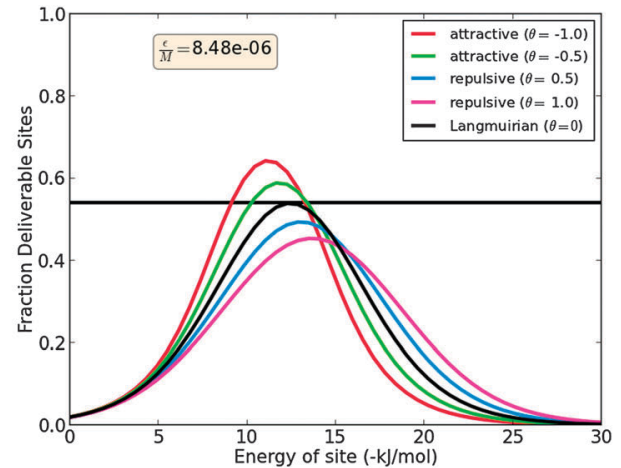

(b)

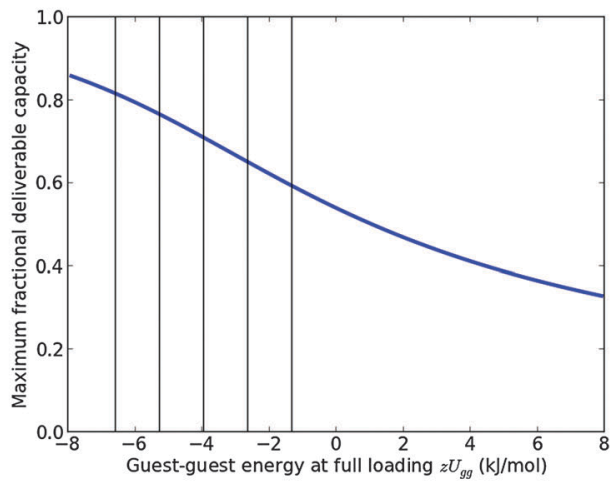

(d)

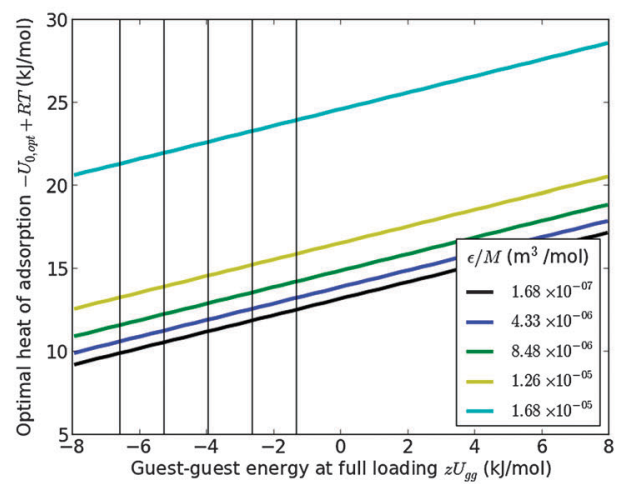

(e)

Fig. 6 Model 1. (a) The influence of guest-guest interactions on the shape of the isotherm in the context of a Langmuirian curve. Depending on where $P_{\mathrm{H}}$ and $P_{\mathrm{L}}$ fall relative to $1 / K$, guest-guest attractions or repulsions may increase the deliverable capacity. Dashed lines are asymptotic approximation in eqn (11). (b) For a given material $\left(\right.$ i.e. $\left.\frac{\varepsilon}{M}\right)$, attractive guest-guest interactions enable a higher maximum fractional deliverable capacity. The horizontal line is the maximum deliverable capacity in eqn (6) for a Langmuirian curve. The optimum energy of the site $-U_{0}$ decreases with more attractive guest-guest interactions ( $\theta$ becoming negative). (c) Again, the optimal infinite dilution heat of adsorption depends on the material (through $\frac{\varepsilon}{M}$ ) with fixed guestguest interactions. The maximum possible fractional deliverable capacity does not depend upon $\frac{\varepsilon}{M}$. Colors of curves based on Fig. 5(a). (d) The maximum fractional deliverable capacity as a function of the guest-guest interaction energy (same curve for all $\frac{\varepsilon}{M}$ ). (e) For a fixed $\frac{\varepsilon}{M}$, the optimal heat of adsorption decreases with attractive guest-guest interactions. Vertical lines in (d) and (e) are multiples of the methane-methane Lennard-Jones parameter in ref. 31 and $32,1.44 \mathrm{~kJ} \mathrm{~mol}^{-1}$.

In Fig. 6(b), we plot the fractional deliverable capacity against the infinite dilution energy of adsorption to show that there still exists a $U_{0}$ that optimizes fractional deliverable capacity. For attractive (repulsive) guest guest interactions $\theta<0(\theta>0)$, the optimum heat of adsorption is below (above) that of a Langmuirian material. Attractive (repulsive) guest-guest interactions yield a larger (smaller) maximum obtainable fractional deliverable capacity than for a Langmuirian isotherm in eqn (6), which is plotted as a horizontal line in Fig. 6(b), consistent with our interpretation of the correction term in eqn (11). Analogous with Fig. 5(b), we show how the optimum $U_{0}$ changes from material to material when there are guest-guest 
interactions in Fig. 6(c) by varying the structural characteristic $\frac{\varepsilon}{M}$. Note that the maximum theoretical deliverable capacity does not change with $\frac{\varepsilon}{M}$. We numerically solve for the maximum theoretical fractional deliverable capacity as a function of guest-guest interaction strength in Fig. 6(d) to provide an analog of eqn (6). In Fig. 6(e), we show how the optimal heat of adsorption decreases with attractive guest-guest interactions. Fig. 6(e) serves as a reference to, for a given pore volume $\left(\frac{\varepsilon}{M}\right)$ and pocket-to-pocket distances and coordination number $\left(\rightarrow z U_{\text {gg }}\right)$, determine the optimal heat of adsorption. Each vertical line is a multiple of the Lennard-Jones interaction parameter for methane using the TraPPE force field ${ }^{40}$ to provide a relevant reference for guest-guest interaction energies.

We can understand the reason that the optimal heat of adsorption for attractive guest-guest interactions is below that of a Langmuirian curve. For a Langmuirian curve, a high $-U_{0}$ ensures that loading is close to $M$ at $P_{\mathrm{H}}$, but there is a tradeoff because a large $-U_{0}$ also dictates a high loading at $P_{\mathrm{L}}$, eroding the deliverable capacity. This is the intuition behind why there is an optimum heat of adsorption. The tradeoff shifts towards having a lower $-U_{0}$ when guest-guest interactions are considered in order to give guest-guest interactions the greatest influence at $P_{\mathrm{H}}$ by shifting the pressure at which the isotherm saturates (through $1 / K$ ). Thus, we can lower the value of $-U_{0}$ to

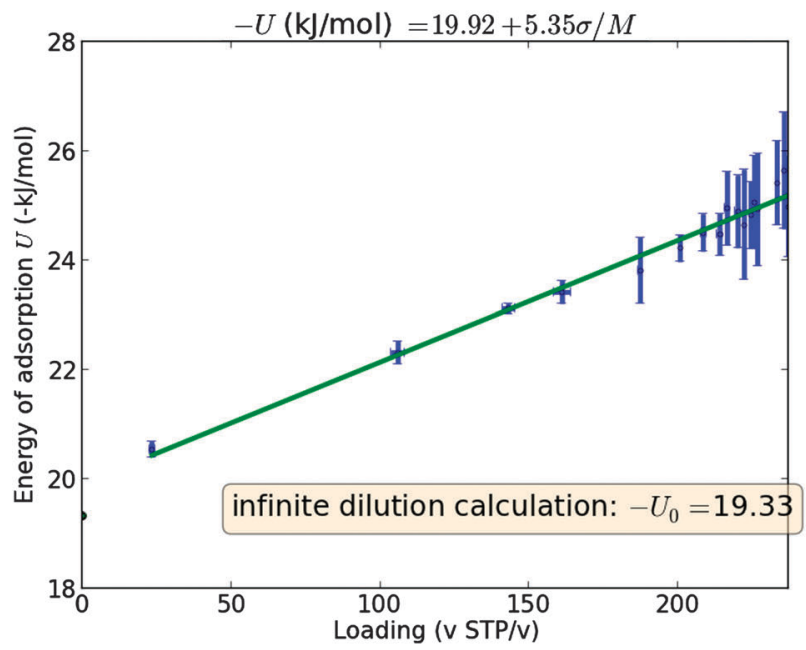

(a)

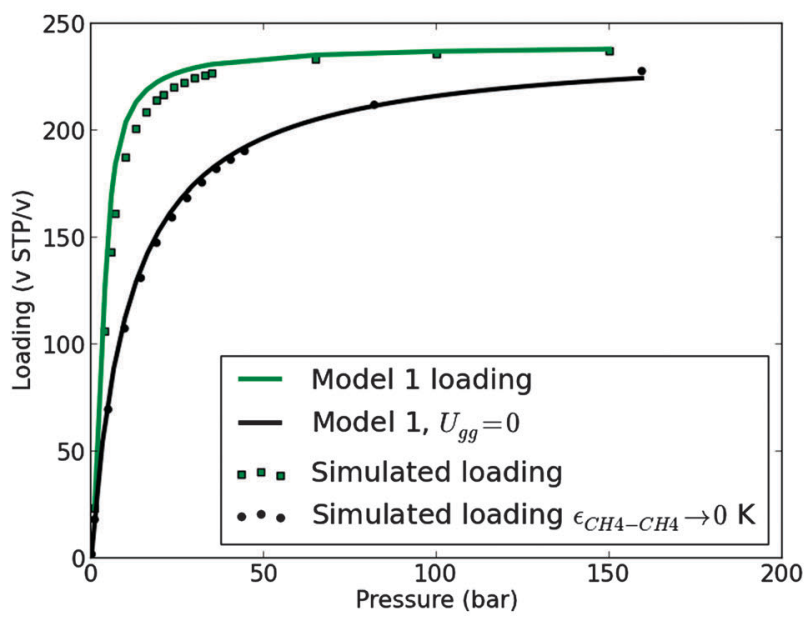

(c)

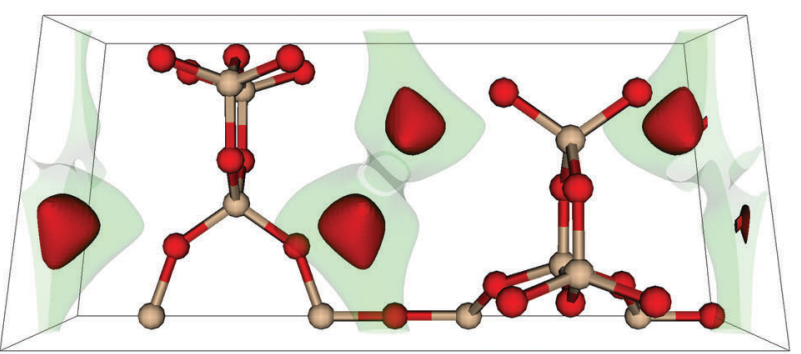

(b)

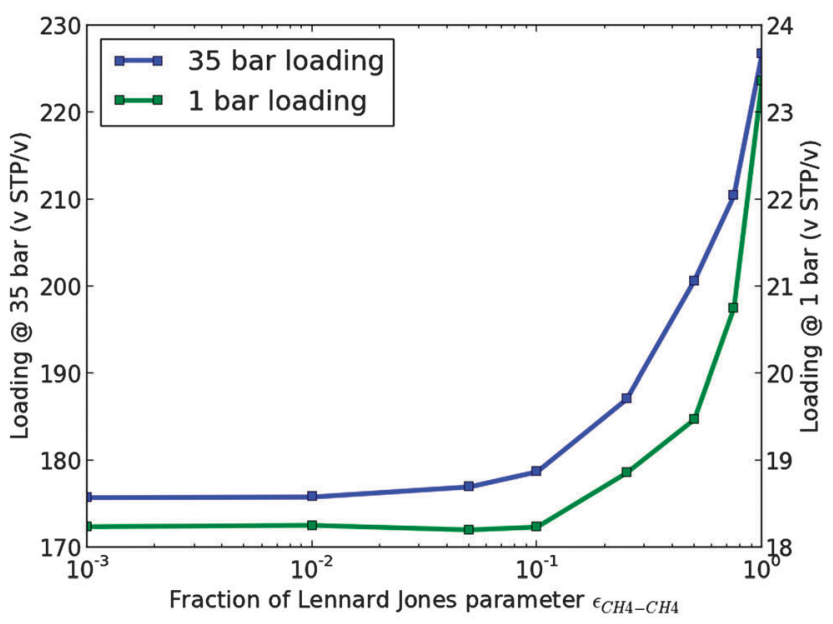

(d)

Fig. 7 SBN has attractive guest-guest interactions. (a) Simulated heat of adsorption increases linearly with loading. We fit to eqn (9). (b) Energy landscape of SBN shows binding sites that are 4 and $4.6 \AA^{41}$ distance from each other, close to the optimal distance of $4.15 \AA$ that maximizes potential overlap for $\mathrm{CH}_{4}-\mathrm{CH}_{4}$ interactions. Red contour: $-2000 \mathrm{~K}$, green contour: $4470 \mathrm{~K}=15 \mathrm{~T}$. (c) $\mathrm{SBN}$ simulated loading as a function of pressure (green

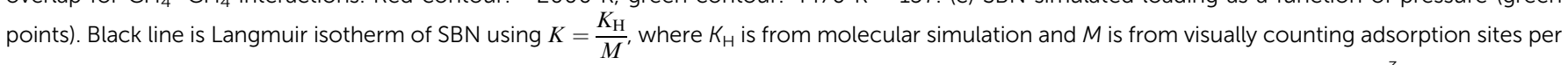

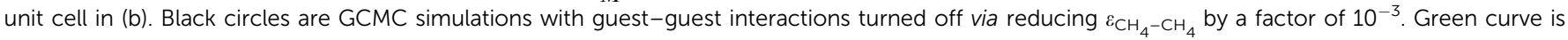

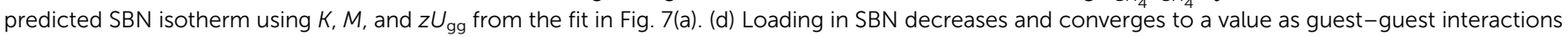
are neglected, simulated by reducing the $\mathrm{CH}_{4}-\mathrm{CH}_{4}$ Lennard-Jones interaction parameter. 
reduce the uptake at $P_{\mathrm{L}}$ and rely on the guest-guest interactions to provide the extra energy to recruit adsorbate molecules at $P_{\mathrm{H}}$.

As an example of beneficial guest-guest attractions, consider the zeolite SBN, which has been identified as a potentially attractive material for methane separations. ${ }^{41}$ In Fig. 7(a), we see that the energy of adsorption of SBN increases linearly with loading, suggesting that attractive guest-guest interactions are present. Fig. 7(b) is a contour plot of the energy landscape of SBN. The binding pockets are $\approx 4$ and $4.6 \AA$ distance from one another, ${ }^{41}$ close to the optimal distance for maximal methanemethane potential overlap of $4.15 \AA$ based on the LennardJones parameter for methane (taken from ref. 31 and 32). From a visual inspection of Fig. 7(b), we observe 4 binding sites in a unit cell of volume $619.36 \AA^{3}$, yielding $M=240 \mathrm{v}_{\mathrm{STP}} / \mathrm{v}$ for SBN. Specified with knowledge of the Henry coefficient and $M$, we plot a Langmuirian SBN isotherm (model $1, U_{\text {gg }}=0$ ) in Fig. $7(\mathrm{c})$ - a thought experiment for when methane-methane interactions are not present. To corroborate this prediction, we 'turn off' guest-guest interactions by letting the Lennard-Jones parameter $\varepsilon$ for $\mathrm{CH}_{4}-\mathrm{CH}_{4}$ interactions go to zero and running GCMC simulations on SBN. We do not set $\varepsilon_{\mathrm{CH}_{4}-\mathrm{CH}_{4}}=0$ because then the feature of excluded volume would be lost; by letting $\varepsilon_{\mathrm{CH}_{4}-\mathrm{CH}_{4}}$ be close to zero, the potential still goes to infinity with two overlapping methane molecules. We plot the loading at 1 bar and 35 bar as a function of guest-guest interaction strength in Fig. 7(d) to show that (i) the loading converges to a particular loading as $\varepsilon_{\mathrm{CH}_{4}-\mathrm{CH}_{4}} \rightarrow 0$ and (ii) guest-guest interactions enhance the loading at 35 bar in comparison to if the SBN isotherm were Langmuirian. Using the expression for $U(\sigma)$ from linear regression in Fig. 7(a), we then predict the actual isotherm of SBN from eqn (10) (model 1), which successfully matches with the simulated loading of SBN in Fig. 7(c).

\subsection{Model 2 and the entropy-enthalpy correlation}

Model 2 presents a different abstraction of a material than in Fig. 3 that captures the coupling between the pore size and the energy of adsorption in the pore. In model 0 , we take the energy of adsorption $U_{0}$ to be independent of the structural characteristics $\varepsilon$ and $M$ of the material. Experimentally, however, a correlation between pore size and heat of adsorption is observed. ${ }^{42,43}$ Ref. 28 adjusted Bhatia and Myers' formula for the optimal heat of adsorption in the context of hydrogen storage by taking into account the positive correlation found between enthalpy and entropy changes upon adsorption. The volume of the adsorption site in model $0, \frac{\varepsilon}{M}$, determines the standard entropy change upon adsorption (ESI, $\dagger$ eqn (S23) and Fig. S4(b)).

For the zeolite database, we calculated the standard entropy change upon adsorption from the computed Henry coefficient and estimated maximum loading (ESI, $\uparrow$ S6.1) and plot it against the computed heat of adsorption in Fig. 8(a). We indeed observe a positive correlation between $|\Delta H|$ and $\left|\Delta S^{\circ}\right|$ up until $\left|\Delta S^{\circ}\right|$ becomes large; at this point, the correlation quickly drops off, an effect not observed in ref. 28. Two properties of the plot in Fig. 8(a) underscore our aforementioned conclusions. First, it is clear from the variance in $\Delta S^{\circ}$ that the entropy change upon adsorption cannot be assumed to be the same for all materials - even within the class of zeolites. Second, Fig. 8(a) is color-coded according to the deliverable capacity; we see that a range of heats of adsorption, between 8 and $23 \mathrm{~kJ} \mathrm{~mol}^{-1}$, yields the zeolites with the highest deliverable capacities, depending upon the associated $\Delta S^{\circ}$. Note that several poor-performing structures are hidden in this image beneath higher-performing structures, highlighting that an optimal heat of adsorption does not guarantee a high deliverable capacity. Similar to ref. 28, we see a barrier entropy change which zeolites do not surpass: around $110 \mathrm{~J} \mathrm{~mol}^{-1} \mathrm{~K}^{-1}$, with a few exceptions shown in the ESI, $\dagger$ S6.3.

We seek to explain the shape of the $|\Delta H|-\left|\Delta S^{\circ}\right|$ correlation observed in Fig. 8(a), by posing a simple model that captures the coupling between the size of the adsorption site and the energy of the site, both of which are dictated by the placement of framework atoms. We assume that a gas molecule is adsorbed in a spherical cavity of radius $R$ (Fig. 8(b) for the unit cell, see ESI $\dagger$ for details). Inside this cavity, the gas molecules interact with a shell of uniformly distributed oxygen atoms that defines the adsorbent. Given that the gas molecule interacts with the oxygen atoms through a Lennard-Jones potential, an effective potential $U_{\text {eff }}(d ; R)$ that is a function of the guest's distance $d$ from the shell wall was derived in ref. 44 by smearing (performing a surface integration ${ }^{45}$ ) the framework atoms over the spherical cage:

$$
\begin{aligned}
U_{\mathrm{eff}}(d ; R)= & 4 \alpha \varepsilon \frac{2 \pi R}{R-d}\left[\frac{\sigma^{12}}{10}\left(\frac{1}{d^{10}}-\frac{1}{(2 R-d)^{10}}\right)\right. \\
& \left.-\frac{\sigma^{6}}{4}\left(\frac{1}{d^{4}}-\frac{1}{(2 R-d)^{4}}\right)\right] .
\end{aligned}
$$

We use the oxygen-methane Lennard-Jones parameters ${ }^{31,32}$ for $\varepsilon$ and $\sigma$ in eqn (12). The parameter $\alpha$ is the surface density of the oxygen atoms in the spherical shell, which we take as $0.13 \AA^{-2}$ by fitting to the maximum enthalpy change in Fig. 8(a).

By varying the radius of the spherical shell, we simultaneously change the standard entropy loss upon adsorption (the "size" of the site) and the enthlapy upon adsorption (through the energy potential). Fig. 8(c) shows how the potential inside the spherical shell changes as a function of the radius $R$. For large radii, adsorbed methane molecules will prefer to reside near the sphere surface, where the potential is deepest. The potential gets deeper as the radius decreases, and methane begins to interact with a greater fraction of the framework atoms in the cage. Eventually, the potential well is deepest in the center of the sphere, the point where methane maximizes its interactions with all framework atoms in the cage. As the radius gets too small, however, repulsive forces take effect, and the potential becomes positive. This repulsive effect is the source of the dropoff in Fig. 8(a).

We now assume that our material consists of these spherical shells packed in a cubic lattice at a shell-to-shell distance $x=$ $2^{1 / 6} \sigma_{\mathrm{O}-\mathrm{O}}$. We compute for spheres of different radii $R$ the Henry coefficient and ensemble-average energy of adsorption using 


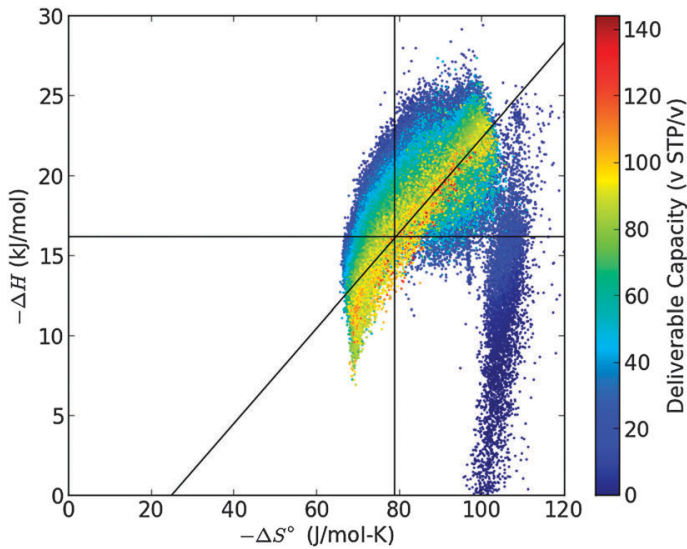

(a)

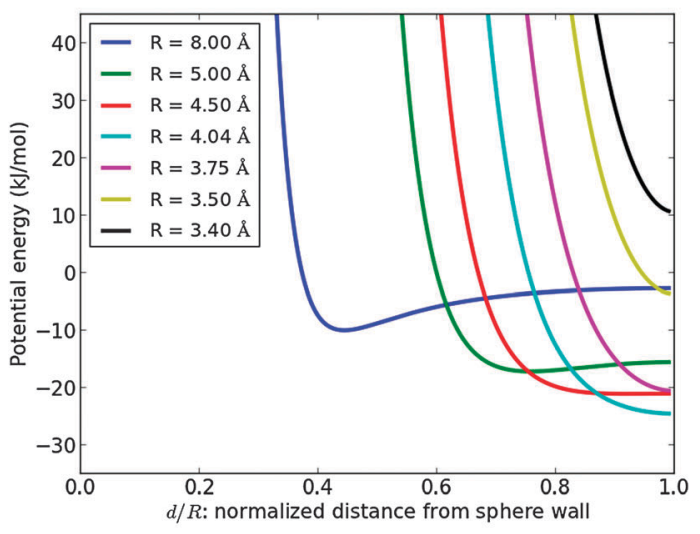

(c)

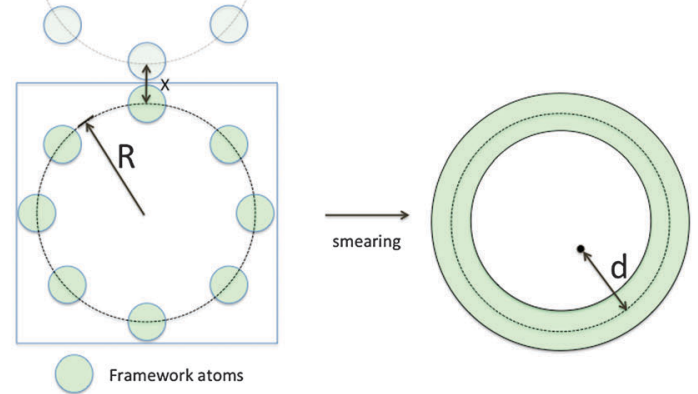

(b)

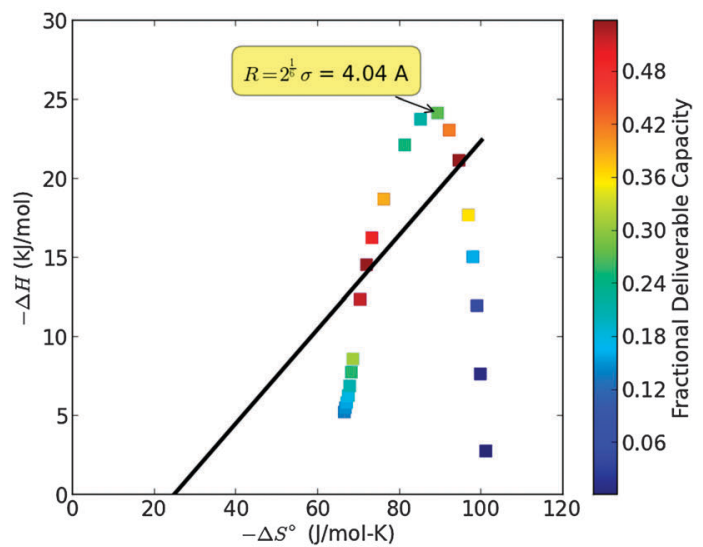

(d)

Fig. 8 Entropy-enthalpy correlation. (a) Correlation between enthalpy and entropy change upon adsorption calculated for the zeolite database. Points, which represent structures, are color-coded according to the deliverable capacity. The black diagonal is the optimal heat of adsorption a function of $\Delta S^{\circ}$ in eqn (5); horizontal line is the blanket $16.2 \mathrm{~kJ} \mathrm{~mol}^{-1}$ optimal heat of adsorption for all materials from using the assumption $\Delta S^{\circ}=-9.5 R$ (vertical line) in ref. 25 , that the standard entropy change upon adsorption is the same for all materials. The structures with the highest deliverable capacity were plotted last, so there are many poor performing structures buried by the high performance structures. (b) A 2D representation of our toy model to understand the entropy-enthalpy correlation. A spherical cage of framework atoms with radius $R$ creates the adsorption pocket inside. The square is the unit cell. The potential $U_{\text {eff }}(d ; R)$ smears the framework atoms over the spherical shell. The variable $d$ is the closest distance from a point to the shell surface. The variable $x$ is the distance between two spherical shells, which we set to be the optimal oxygen-oxygen distance $2^{1 / 6} \sigma_{\mathrm{O}-\mathrm{O}}$. (c) The potential $U_{\text {eff }}(d ; R)$ plotted for a series of sphere radii $R$. (d) The entropy-enthalpy correlation from our toy model mimics that of the zeolite database in (a). Each point corresponds to a simulation of a toy model of a different radius $R$. The radius $R$ decreases monotonically with the entropic loss. The deepest potential well in (c) occurs for $R=\sigma 2^{\frac{1}{6}}$ and yields the largest $|\Delta H|$. The points are color coded according to their fractional deliverable capacity. The optimal heat of adsorption as a function of the standard entropy change in eqn (5) is plotted as the black line.

Widom insertions. To estimate the saturation loading, we compute the helium pore volume and use the density of liquid methane. See ESI, $\uparrow \mathrm{S} 6$ for the details.

Fig. 8(d) shows the coupling between the heat of adsorption and the entropy of adsorption for our model. Our model nicely captures the shape of the corresponding data for the zeolites in Fig. 8(a). We observe a maximum in the heat of adsorption, which corresponds to a pore diameter of $R=R_{\mathrm{c}}:=2^{1 / 6} \sigma$ in which we have the maximum interactions of methane with the spherical shell. If we increase the pore diameter from $R_{\mathrm{c}}$, the heat of adsorption decreases until we reach such a large pore that the effect of the pore walls is negligible. If we, on the other hand, decrease the pore diameter from $R_{\mathrm{c}}$, the interactions are dominated by the repulsive part of the potential, and the magnitude of the enthalpy of adsorption quickly decreases until it becomes positive. The entropy loss upon adsorption increases monotonically with decreasing pore size. The entropy loss in our model has then reached its maximum as the methane molecule is frozen between the wall of the sphere.

In our model, we assumed a cubic lattice packing of spherical shells with distance $x=2^{1 / 6} \sigma_{\mathrm{O}-\mathrm{O}}$. However, the packing can vary, which shifts the $\Delta H-\Delta S^{\circ}$ curve. In addition, the shape of the cavity and surface density of atoms can change, which results in different heats of adsorption. The net effect of these changes will be a collection of curves that each have a similar shape, explaining the thick band we observe in Fig. 8(a). The color coding shows the fractional deliverable capacity for this material. Again, the cage radii with the highest fractional deliverable capacity fall on the line dictated by eqn (5). 
The IZA zeolite structures are plotted in the $\left(\Delta S^{\circ}, \Delta H\right)$ plane along with the hypothetical zeolite structures in Fig. S12 (ESI $\dagger$ ). The IZA structures explore a similarly sizable range of $\Delta S^{\circ}$. As ESI, $\dagger$ we provide an interactive plot of the predicted deliverable capacities of the IZA zeolites in both the 65 to 5.8 bar and 35 to 1 bar pressure ranges.

\section{Analysis of top performing zeolite structures}

It is interesting to compare the results of our models with the characteristics of the top performing structures. Here, we analyze the two zeolites with the largest 35 to 1 bar deliverable capacity, PCOD8124791 and PCOD8330975. PCOD8124791 is topologically identical to the IZA zeolite SBN, however with perturbed oxygen atom positions due to the relaxation step in hypothetical zeolite generation. ${ }^{22}$ These two structures are outliers in the dataset (see Fig. S6, ESI $\dagger$ ) because a combination of factors must arise to, under the constraint of using silica, provide an optimum material. Namely, to resonate with models 0 and 1, the optimal material has a high density of sites, a moderate heat of adsorption, and adsorption pockets positioned for guestguest attractions.

The two top performing materials PCOD8124791 and PCOD8330975 have predicted 35 to 1 bar deliverable capacities of $200 \mathrm{v}_{\mathrm{STP}} / \mathrm{v}$ and $172 \mathrm{v}_{\mathrm{STP}} / \mathrm{v}$, respectively. Satisfying the first prerequisite, they both display a high density of binding sites, obtained by counting the adsorption pockets per unit cell from Fig. 9, corresponding to $M=245 \mathrm{v}_{\mathrm{STP}} / \mathrm{v}$ and $M=221 \mathrm{v}_{\mathrm{STP}} / \mathrm{v}$, respectively. PCOD8330975 has a $10 \%$ lower density of sites due to the cubic lattice-like arrangement compared to the more efficient site packing in PCOD8124791, resulting in reduced performance. This corresponds to an $82 \%$ and $78 \%$, respectively, utilization of sites to deliver a methane molecule - significantly higher than the $70 \%$ theoretical maximum utilization by eqn (6) for a Langmuirian material. The pairwise distances between sites

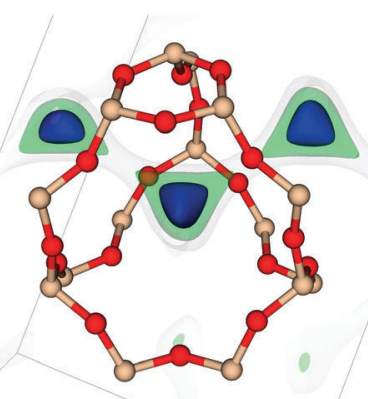

(a)

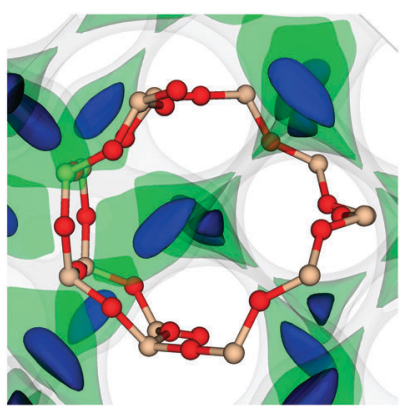

(b)
Fig. 9 Visualization of the structure unit cell and energy landscape of the top performing zeolites. Contours in the potential surfaces correspond to energies of $-2000 \mathrm{~K}$ (blue, the strongest binding regions), $0 \mathrm{~K}$ (green), and $4470 \mathrm{~K}$ (gray, representing the channel system defined approximately as 15T). Silicon atoms are shown in tan; oxygen red. (a) First place deliverable capacity material, PCOD8124791 (b) second place deliverable capacity material, PCOD8330975. in zeolites PCOD8124791 and PCOD8330975 are near the optimal Lennard-Jones distance for maximizing potential overlap ( $\sim 4.15 \AA$, see Fig. 9), leading to attractive guest-guest interactions and enabling these materials to utilize a greater fraction of their sites to deliver a molecule to the tank/fuel cell. Indeed, the simulated heats of adsorption of PCOD8124791 and PCOD8330975 increase with loading (Fig. S11, ESI $\dagger$ ). This resonates with the story of Fig. 6(d), where guest-guest attractions were shown to increase the fraction of sites that are utilized to deliver a methane molecule to the engine beyond that possible with a Langmuirian material. We found that zeolites perform better in the 35 to 1 bar pressure range operation than in the 65 to 5.8 bar operation because their isotherms are generally steep at low pressures (1-5.8 bar).

For attractive guest-guest attractions to benefit the deliverable capacity, the infinite dilution heat of adsorption must be at the respective optimum to ensure that the isotherm saturates at the correct point with respect to $P_{\mathrm{L}}$ and $P_{\mathrm{H}}$. Many other structures have heats of adsorption that increase with loading and thus attractive guest-guest interactions, but are not outlying performers. That is, $P_{\mathrm{L}}$ must be low enough in the isotherm so that the guest-guest attractions do not enhance uptake at $P_{\mathrm{L}}$ and degrade the deliverable capacity (Fig. S3(a), ESI $\dagger$ ).

The methane binding sites in PCOD8124791 and PCOD8330975 arise from similar local atomic arrangments in their structures. Biding sites in PCOD8124791 are comprised of three 8-Si rings (see Fig. 9(a)), which form the apertures in the channel system; these are capped by a 3-Si ring (top in figure) and a 9-Si ring (bottom), each of which is too small to form an aperture for methane. In PCOD8330975 (see Fig. 9(b)), we see the same 8-Si aperture shape, but the ring systems differ: three 4-Si rings cap the binding site (left), while the apertures comprise 10-Si rings (due to the two additional Si seen at figure right which do not constitute part of the 8-Si shape). Accordingly, the atoms contributing to the creation of the binding site in these two materials are strikingly similar: $15 \mathrm{Si}$ and $18 \mathrm{O}$, and $14 \mathrm{Si}$ and $19 \mathrm{O}$, respectively (note these atoms are shared between multiple binding sites). In previous work, ${ }^{46,47}$ we identified these 8-Si shapes for achieving favorable binding sites in zeolites for small hydrocarbon and $\mathrm{CO}_{2}$ guest molecules; here we show that these features also yield optimal methane binding pockets in zeolites.

Some materials do not have defined sites such as in model 0 , but rather large, open surface areas of framework atoms that attract guest molecules. Bhatia and Myers showed that the optimal material will not have heterogeneous sites, which is intuitive because "it is the optimal site that is most productive". ${ }^{25}$ We see this in the top-performing zeolites PCOD8124791 and PCOD8330975, whose adsorption pockets are relatively homogeneous and do not have large cavities (which will certainly have regions of suboptimal energies) that provide open surface areas for adsorption.

There are no known synthesis routes to obtain any of the given hypothetical zeolites that we identified as top performers. However, advancements in the design of organic structure-directing agents $^{48}$ may change this situation. For example, a recent work 
by the Deem group ${ }^{49}$ demonstrates a prototype approach for such a design.

\section{Discussion}

In this work, we have extended the ideas developed by Bhatia and Myers ${ }^{25}$ concerning an optimum heat of adsorption for methane storage in nanoporous materials using molecular simulation results from the IZA and hypothetical zeolite databases and thermodynamic models as guides. In the context of a Langmuir isotherm, there exists a heat of adsorption that maximizes the fraction of adsorption sites that will deliver a methane molecule to the engine. An optimal heat of adsorption does not, however, guarantee that a material will exhibit a large deliverable capacity (Fig. 2) since this depends on the density of adsorption sites. Moreover, our analysis has shown that this optimal heat of adsorption is material-dependent because of differing entropy changes upon adsorption, and hence there does not exist a blanket optimal heat of adsorption target for all materials.

Our sequence of models manifests features that endow a material with a large deliverable capacity. The first prerequisite is that a material must have a high density of sites, as this is an upper-bound for the deliverable capacity. Given a density of sites, designing an optimal material reduces to optimizing the fraction of sites utilized to deliver a guest. If the number of adsorption sites per volume multiplied by the maximum deliverable fraction of sites (eqn (6) or Fig. 6(d))) is less than the target deliverable capacity, the material cannot possibly reach the target. To maximize the fractional deliverable capacity, one would ideally substitute chemical moieties to effectively tune the infinite dilution heat of adsorption, using our model as a guide (eqn (4) or Fig. 6(e)). One must consider that, if differently sized chemical moieties are substituted, this changes the entropic loss upon adsorption (via $\frac{\varepsilon}{M}$ ), and thus the optimal heat of adsorption shifts from what it was at the starting point.

Wilmer et $a .^{24}$ found that, in analyzing a large set of hypothetical MOFs, the materials with the highest deliverable capacities had a void fraction of $\sim 0.8$. The zeolites analyzed in this work exhibit void fractions (calculated from Zeo++ with a methane probe of $1.625 \AA$ radius) in the range $\sim[0,0.5]$. Because materials with more adsorption sites per volume will generally have higher void fractions (correlation in Fig. 5(a)), this suggests that zeolites have a smaller density of sites, rendering them, on average, poorer materials for gas storage. Zeolite are relatively dense nanoporous materials, thus their gravimetric deliverable capacities are generally poorer compared to the MOFs considered in ref. 24.

The heat of adsorption is generally a function of the loading. Attractive guest-guest interactions cause the energy of adsorption to become more negative with loading. The distance between two methane molecules that maximizes attractive energies is $\sim 4.15 \AA$ (based on both ref. 31 and 32 and TraPPE ${ }^{40}$ ). Model 1 shows that, if the infinite dilution heat of adsorption is at an optimal value (see Fig. 6(d)), attractive guest-guest interactions can substantially enhance the fractional deliverable capacity beyond that of a Langmuirian material. Ideally, one would like to have control over the positioning of the adsorption pockets such that they are $\sim 4.15 \AA$ apart. The deliverable capacity of the most promising zeolites, SBN, PCOD8124791, and PCOD8330975 among them, is enhanced by having their pockets positioned at the optimal distance for attractive guest-guest interactions. Note, however, that if the infinite dilution heat of adsorption is too high, guest-guest repulsions will increase the fractional deliverable capacity (Fig. 6(b)) by reducing uptake at $P_{\mathrm{L}}$.

The void fraction can help distinguish the deliverable capacity of materials with the same heat of adsorption in Fig. 2 precisely because of the $\varepsilon-M$ correlation in Fig. 5(a): more porous materials will have a higher density of sites. The story of Fig. 2 is that, to obtain a material with a high deliverable capacity, first maximize the density of sites (related to void fraction), and then optimize the heat of adsorption.

With our model in Fig. 8(b), we intuitively highlight how the entropy and enthalpy change upon adsorption are coupled. Model 2 explains the enthalpy-entropy correlation reported in ref. 28: the largest entropy changes are for small adsorption sites, which will have framework atoms in close proximity to the adsorbed methane. These proximal framework atoms will provide potential overlap to make the energy of adsorption more negative. As the site gets too small, however, repulsion takes effect and the enthlapy change quickly drops off as the site gets smaller. In this regime, structures will have very low guest loading. The reason the dropoff in Fig. 8(a) was not observed in ref. 28 is that they did not consider very poorly performing structures in their dataset.

Düren et al. ${ }^{15}$ focus on the problem of obtaining the largest methane uptake in a material at the charging pressure $P_{\mathrm{H}}$, assuming that the gas tanks are designed in such a way that we can use all methane adsorbed for the combustion. With this criteria, the best material must have a large accessible surface area and free volume and strong energetic interactions with adsorbed methane. They note that it is not straightforward to tune these properties by changing the pore size because, "to improve one of these may worsen the others in a complex manner". The model in which we vary the radius of the spherical shell of framework atoms that build an adsorption pocket (model 2) quantitatively elucidates why: changing the pore size affects the energy landscape inside the pore in addition to the free volume for methane occupancy.

The free energy landscape ultimately dictates the deliverable capacity of methane in a material. Correlations with surface areas and pore sizes such as in Fig. 1(c) and (d) attempt to distill a complicated thermodynamic problem into simple, onedimensional, guidelines, but should be taken with caution. One cannot always extrapolate geometric correlations with deliverable capacity far beyond the $298 \mathrm{~K}, 65$ to 5.8 bar pressure range and the space of non-silica nanoporous materials. As an example, correlations of uptake with pore size and accessible surface area look different for different pressures (Fig. S7 and S8, ESI $\dagger$ ). Model 2 shows that the best pore size, even under the assumption of a spherical adsorption pocket, 
depends upon the atoms used to build the adsorption pocket and the surface density of those atoms; zeolites will have a different optimal pore size than, for example, MOFs. Futher, we cannot be sure that the zeolite database, despite its vastness, exhausts the complete "space" of materials for a comprehensive analysis. That is, spurious relationships can arise from the fact that only a subset of possible materials were analyzed.

In summary, the potential for adsorbed natural gas technology is promising, and we hope our conclusions from the analysis of the zeolite dataset and our models help the materials-synthesis community intuit what features endow a material with a high deliverable capacity to guide in designing the optimum material. Finally, it is important to note that our conclusions can be applied to storing other gases in nanoporous materials, such as hydrogen. ${ }^{50}$ Subject to the storage condition, one can perform a similar analysis as in this study.

\section{Acknowledgements}

Thanks to Lev Sarkisov for statistical mechanics discussions and Stack Overflow for answering plotting questions. This work is supported by the U.S. Department of Energy, Office of Basic Energy Sciences, Division of Chemical Sciences, Geosciences and Biosciences under Award DE-FG02-12ER16362. This research used resources of the National Energy Research Scientific Computing Center, which is supported by the Office of Science of the U.S. Department of Energy under Contract No. DE-AC02-05CH11231.

\section{References}

1 K. Konstas, T. Osl, Y. Yang, M. Batten, N. Burke, A. J. Hill and M. R. Hill, J. Mater. Chem., 2012, 22, 16698-16708.

2 U.S. Department of Energy Energy Information Administration, 2013, http://www.eia.gov/tools/faqs/faq.cfm?id=73t=11.

3 U.S. E.P.A., 2013, http://www.epa.gov/climatechange/Downloads/ ghgemissions/US-GHG-Inventory-2013-ES.pdf.

4 U.S. Dept. of Energy - Argonne National Laboratory Report: A Full Fuel-Cycle Analysis of Energy and Emissions Impacts of Transportation Fuels Produced from Natural Gas, 12/1999.

5 MIT, 2011, http://mitei.mit.edu/publications/reports-studies/ future-natural-gas.

6 R. A. Alvarez, S. W. Pacala, J. J. Winebrake, W. L. Chameides and S. P. Hamburg, Proc. Natl. Acad. Sci. U. S. A., 2012, 109, 6435-6440.

7 A. P. Pacsi, N. S. Alhajeri, D. Zavala-Araiza, M. D. Webster and D. T. Allen, Environ. Sci. Technol., 2013, 47, 3521-3527.

8 S. G. Osborn, A. Vengosh, N. R. Warner and R. B. Jackson, Proc. Natl. Acad. Sci. U. S. A., 2011, 108, 8172-8176.

9 Y. F. Makogon, J. Nat. Gas Sci. Eng., 2010, 2, 49-59.

10 N. Bolan, R. Thangarajan, B. Seshadri, U. Jena, K. Das, H. Wang and R. Naidu, Bioresour. Technol., 2013, 135, 578-587.
11 A. D. Cullar and M. E. Webber, Environ. Res. Lett., 2008, 3, 034002.

12 T. A. Makal, J.-R. Li, W. Lu and H.-C. Zhou, Chem. Soc. Rev., 2012, 41, 7761-7779.

13 Y. Peng, V. Krungleviciute, I. Eryazici, J. T. Hupp, O. K. Farha and T. Yildirim, J. Am. Chem. Soc., 2013, 135, 11887-11894.

14 C. E. Wilmer, O. K. Farha, T. Yildirim, I. Eryazici, V. Krungleviciute, A. A. Sarjeant, R. Q. Snurr and J. T. Hupp, Energy Environ. Sci., 2013, 6, 1158-1163.

15 T. Düren, L. Sarkisov, O. M. Yaghi and R. Q. Snurr, Langmuir, 2004, 20, 2683-2689.

16 M. Eddaoudi, J. Kim, N. Rosi, D. Vodak, J. Wachter, M. O'Keeffe and O. M. Yaghi, Science, 2002, 295, 469-472.

17 H. Furukawa and O. M. Yaghi, J. Am. Chem. Soc., 2009, 131, 8875-8883.

18 R. B. Getman, Y.-S. Bae, C. E. Wilmer and R. Q. Snurr, Chem. Rev., 2011, 112, 703-723.

19 Advanced Research Projects Agency - Energy, US Department of Energy, 2012, http://arpa-e.energy.gov/?q=arpa-e-sitepage/view-programs.

20 J. Mota, A. Rodrigues, E. Saatdjian and D. Tondeur, Adsorption, 1996, 3, 117-125.

21 J. A. Mason, M. Veenstra and J. R. Long, Chem. Sci., 2014, 5, 32-51.

22 M. W. Deem, R. Pophale, P. A. Cheeseman and D. J. Earl, J. Phys. Chem. C, 2009, 113, 21353-21360.

23 R. Pophale, P. A. Cheeseman and M. W. Deem, Phys. Chem. Chem. Phys., 2011, 13, 12407-12412.

24 C. E. Wilmer, M. Leaf, C. Lee, O. K. Farha, B. G. Hauser, J. T. Hupp and R. Q. Snurr, Nat. Chem., 2011, 4, 83-89.

25 S. K. Bhatia and A. L. Myers, Langmuir, 2006, 22, 1688-1700.

26 Y.-S. Bae and R. Q. Snurr, Microporous Mesoporous Mater., 2010, 132, 300-303.

27 R. B. Getman, J. H. Miller, K. Wang and R. Q. Snurr, J. Phys. Chem. C, 2011, 115, 2066-2075.

28 E. Garrone, B. Bonelli and C. O. Aren, Chem. Phys. Lett., 2008, 456, 68-70.

29 T. F. Willems, C. H. Rycroft, M. Kazi, J. C. Meza and M. Haranczyk, Microporous Mesoporous Mater., 2012, 149, 134-141.

30 D. Frenkel and B. Smit, Understanding molecular simulation: From algorithms to applications, 1996.

31 D. Dubbeldam, S. Calero, T. Vlugt, R. Krishna, T. L. Maesen, E. Beerdsen and B. Smit, Phys. Rev. Lett., 2004, 93, 088302.

32 S. Calero, D. Dubbeldam, R. Krishna, B. Smit, T. J. Vlugt, J. F. Denayer, J. A. Martens and T. L. Maesen, J. Am. Chem. Soc., 2004, 126, 11377-11386.

33 R. L. Martin, D. D. Donofrio, J. A. Sethian and M. Haranczyk, et al., Int. J. High Perform. Comput. Appl., 2012, 26, 347-357.

34 J. Kim, R. L. Martin, O. Rubel, M. Haranczyk and B. Smit, J. Chem. Theory Comput., 2012, 8, 1684-1693.

35 S. Sircar, R. Mohr, C. Ristic and M. B. Rao, J. Phys. Chem. B, 1999, 103, 6539-6546. 
36 R. L. Martin, B. Smit and M. Haranczyk, J. Chem. Inf. Model., 2012, 52, 308-318.

37 V. Menon and S. Komarneni, J. Porous Mater., 1998, 5, 43-58.

38 A. L. Myers, Colloids Surf., A, 2004, 241, 9-14.

39 K. R. Matranga, A. L. Myers and E. D. Glandt, Chem. Eng. Sci., 1992, 47, 1569-1579.

40 M. G. Martin and J. I. Siepmann, J. Phys. Chem. B, 1998, 102, 2569-2577.

41 J. Kim, A. Maiti, L.-C. Lin, J. K. Stolaroff, B. Smit and R. D. Aines, Nat. Commun., 2013, 4, 1694.

42 B. Schmitz, U. Müller, N. Trukhan, M. Schubert, G. Férey and M. Hirscher, ChemPhysChem, 2008, 9, 2181-2184.

43 S. Tedds, A. Walton, D. P. Broom and D. Book, Faraday Discuss., 2011, 151, 75.
44 J. Ripmeester and C. Ratcliffe, J. Phys. Chem., 1990, 94, $7652-7656$.

45 B. Cox, N. Thamwattana and J. Hill, Proc. R. Soc. London, Ser. A, 2007, 463, 477-494.

46 J. Kim, L.-C. Lin, R. L. Martin, J. A. Swisher, M. Haranczyk and B. Smit, Langmuir, 2012, 28, 11914-11919.

47 R. Martin, T. Willems, L. Lin, J. Kim, J. Swisher, B. Smit and M. Haranczyk, ChemPhysChem, 2012, 13, 3595-3597.

48 R. F. Lobo, S. I. Zones and M. E. Davis, J. Inclusion Phenom. Mol. Recognit. Chem., 1995, 21, 47-78.

49 R. Pophale, F. Daeyaert and M. W. Deem, J. Mater. Chem. A, 2013, 1, 6750-6760.

50 L. J. Murray, M. Dinca and J. R. Long, Chem. Soc. Rev., 2009, 38, 1294-1314. 